\title{
Global Small data Solutions for a system of semilinear heat equations and the corresponding system of damped wave equations with nonlinear memory
}

\section{Solutions globales pour un système d'équations de chaleur semi-linéaires et système correspondant d'équations d'ondes amorties}

\author{
Mohamed Berbiche ${ }^{1}$ and Messaouda Terchi ${ }^{2}$ \\ ${ }^{1}$ Laboratory of Mathematical Analysis, Probability and Optimizations, \\ University of Biskra, Po. Box 145, Biskra (07000), Algeria \\ mohamed.berbiche@univ-biskra.dz,berbichemed@yahoo.fr \\ ${ }^{2}$ Laboratory of Mathematical Analysis, Probability and Optimizations, \\ University of Biskra, Po. Box 145, Biskra (07000), Algeria \\ terchi.messaouda@gmail.com
}

\begin{abstract}
We consider the Cauchy problem for a strongly coupled semi-linear heat equations with some kind of nonlinearity in multi-dimensional space $\mathbb{R}^{N}$. We see under some conditions on the exponents and on the dimension $N$, that the existence and uniqueness of time-global solutions for small data and their asymptotic behaviors are obtained. This observation will be applied to the corresponding system of the damped wave equations in low dimensional space.

Mathematics Subject Classification 2010: 35K45, 35A01, 35B33

KEYWORDS. Parabolic system, damped wave system, global existence, critical exponent
\end{abstract}

\section{Introduction}

We consider the Cauchy problem for a system of strongly coupled semilinear integro-differential equations of parabolic type

$$
\left\{\begin{array}{l}
u_{t}-\Delta u=\int_{0}^{t}(t-s)^{-\gamma_{1}}|u(s)|^{p_{1}}|v(s)|^{q_{1}} d s, t>0, x \in \mathbb{R}^{N}, \\
v_{t}-\Delta v=\int_{0}^{t}(t-s)^{-\gamma_{2}}|v(s)|^{p_{2}}|u(s)|^{q_{2}} d s, t>0, x \in \mathbb{R}^{N}, \\
u(0, x)=u_{0}(x), v(0, x)=v_{0}(x), x \in \mathbb{R}^{N},
\end{array}\right.
$$

where the unknown functions $u:=u(t, x), v:=v(t, x)$ are real-valued, $N \geq 1, \Delta$ is the Laplacian, $p_{1}, q_{1}, p_{2}, q_{2} \geq 1,0<\gamma_{1}, \gamma_{2}<1$ and $u_{0}(x), v_{0}(x)$ are the given initial data.

It is well known that the nonlinear source terms in (1.1) can be expressed in terms of Riemann-Liouville fractional integral operator which is defined by

$$
J_{a \mid t}^{\alpha} g(t):=\frac{1}{\Gamma(\alpha)} \int_{a}^{t}(t-s)^{\alpha-1} g(s) d s
$$


with $a=-\infty$ by Liouville in 1832 and with $a=0$ by Riemann in 1876 (see Chapter V in [9]), where $\Gamma(\alpha)$ is Euler Gamma function. Since the limit

$$
\lim _{\gamma \rightarrow 1} \frac{1}{\Gamma(1-\gamma)} s_{+}^{-\gamma}=\delta(s)
$$

exists in distribution sense, where $s_{+}:=\max (0, s), \delta(s)$ is the Dirac function. The nonlinear interaction in Cauchy problem (1.1) can be viewed as approximations of classical semilinear parabolic systems when $\gamma_{1}, \gamma_{2}$ converge to 1 .

In recent years, the semi-linear Volterra diffusion equations have been studied extensively in the literature, since they appear naturally in problems involving population dynamics [37, 38], compression of poro-viscoelastic media [13], thermodynamics of phase transition [7], reaction-diffusion problems [11] and nuclear reactor kinetics $[19,30]$.

There is a wide literature on the qualitative properties of solutions to the heat equations and the damped wave equations with polynomial nonlinearities, see for example, [2]-[44], and the references therein. These works deal with the questions of global existence, asymptotic behavior, blow-up in finite time and so forth as well as a variety of methods used to study these questions.

On the other hand, it seems that there are a few results related to the problems (1.1) with time nonlocal nonlinear source as compared with nonlinear polynomial source (see for instance ([3],[22],[1],[42],[39], [40],[4],[5],[41]). Here we only mention some of them motivate our work.

In [3], Cazenave et al. studied the global existence and blow-up for the parabolic equation with nonlocal in time non-linearity

$$
\partial_{t} u(t, x)-\Delta u(t, x)=\int_{0}^{t}(t-\tau)^{-\gamma}|u|^{p-1} u(\tau, x) d \tau,
$$

where $0 \leq \gamma<1, p>1$ and $u_{0} \in C_{0}\left(\mathbb{R}^{N}\right)$. They showed that the Fujita critical exponent is not the one predicted from the scaling argument, which is different from the heat equation.

More precisely they proved that, if

$$
p_{\gamma}=1+\frac{2(2-\gamma)}{(n-2+2 \gamma)_{+}}
$$

and $p_{*}=\max \left\{\frac{1}{\gamma}, p_{\gamma}\right\} \in(0,+\infty]$, where $(u)_{+}=\max (u, 0)$, then:

(i) If $\gamma \neq 0, p \leq p_{*}$, and $u_{0} \geq 0, u_{0} \neq \equiv$, then $u$ blows up in finite time.

(ii) If $\gamma \neq 0, p>p_{*}$, and $u_{0} \in L_{q_{s c}}\left(\mathbb{R}^{N}\right)$ (where $q_{s c}=N(p-1) /(4-2 \gamma)$ ) with $\left\|u_{0}\right\|_{L_{q_{s c}}}$ sufficiently small, then $u$ exists globally.

This result is later extended by some authors to the weakly coupled parabolic systems, damped wave equations, weakly coupled damped wave systems, we refer the reader to [22, 42, 39, 5, 4, 40, 41, 1, 44]. We just briefly describe the results directly connected to our problems. 
In Loayza et al. [22] discussed the following weakly coupled nonlocal coupled parabolic system

$$
\left\{\begin{array}{l}
u_{t}-\Delta u=\int_{0}^{t}(t-s)^{-\gamma_{1}}|v(s)|^{p} d s, t>0, x \in \Omega \\
v_{t}-\Delta v=\int_{0}^{t}(t-s)^{-\gamma_{2}}|u(s)|^{q} d s, t>0, x \in \Omega \\
u(0, x)=u_{0}(x), v(0, x)=v_{0}(x), x \in \Omega
\end{array}\right.
$$

with $p, q \geq 1,0 \leq \gamma_{1}, \gamma_{2}<1, \Omega \subset \mathbb{R}^{N}$ is a bounded domain with smooth boundary or $\Omega=\mathbb{R}^{N}$ and $u_{0}$, $v_{0} \in C_{0}\left(\mathbb{R}^{N}\right)$. They established the following: Assume $p, q \geq 1$ such that $p q>1$

(i) if $\left\{\begin{array}{l}\frac{N}{2}(p q-1)>\left(1-p \gamma_{2}\right)+p\left(1-q \gamma_{1}\right)+p(q+1), \\ \frac{N}{2}(p q-1)>\left(1-q \gamma_{1}\right)+q\left(1-p \gamma_{2}\right)+q(p+1),\end{array}\right.$

(ii) if $\left\{\begin{array}{l}1-p \gamma_{2}+p\left(1-q \gamma_{1}\right)<0 \\ 1-q \gamma_{1}+q\left(1-p \gamma_{2}\right)<0\end{array}\right.$

(iii) and if $\left(\frac{p}{r_{2}}-\frac{2}{N}\right)<\frac{1}{q},\left(\frac{q}{r_{1}}-\frac{2}{N}\right)<\frac{1}{p}$, with $r_{1}=\frac{N(p q-1)}{2\left[2-\gamma_{1}+p\left(2-\gamma_{2}\right)\right]}, \quad r_{2}=\frac{N(p q-1)}{2\left[2-\gamma_{2}+q\left(2-\gamma_{1}\right)\right]}$. Then global solutions with small initial data exist.

Whereas if

(i) $\left\{\begin{array}{l}\frac{N}{2}(p q-1) \leq\left(1-p \gamma_{2}\right)+p\left(1-q \gamma_{1}\right)+p(q+1) \text {, or } \\ \frac{N}{2}(p q-1) \leq\left(1-q \gamma_{1}\right)+q\left(1-p \gamma_{2}\right)+q(p+1) \text {, or }\end{array}\right.$,

(ii) $1-p \gamma_{2}+p\left(1-q \gamma_{1}\right) \geq 0$, or $1-q \gamma_{1}+q\left(1-p \gamma_{2}\right) \geq 0$,

then every nontrivial solution blows up in finite time.

A natural generalization of classical weakly coupled system (1.1) are corresponding damped wave system

$$
\left\{\begin{array}{l}
u_{t t}-\Delta u+u_{t}=\int_{0}^{t}(t-s)^{-\gamma_{1}}|v(s)|^{p} d s, t>0, x \in \mathbb{R}^{N}, \\
v_{t t}-\Delta v+v_{t}=\int_{0}^{t}(t-s)^{-\gamma_{2}}|u(s)|^{q} d s, t>0, x \in \mathbb{R}^{N}, \\
u(0, x)=u_{0}(x), v(0, x)=v_{0}(x), u_{t}(0, x)=u_{1}(x), v_{t}(0, x)=v_{1}(x) x \in \mathbb{R}^{N} .
\end{array}\right.
$$

In [40], Xu considered the problem (1.3), he proved global existence and asymptotic behavior as $t \rightarrow \infty$ of small data solutions in the case when $N=1$, also, he showed under some positive data nonexistence of nonnegative weak solutions for $N \geq 1$. The method used in [40] is inspired from the weighted energy method developed by Todorova and Yordanov [36]. As we have seen, Xu restricts himself in the case of compactly supported data and the dimension $N=1$. 
Recently Berbiche [4], studied the problem (1.3), he obtained the small data global solution result in low-dimensional space $1 \leq N \leq 3$ with noncompactly supported initial data and obtained the $L^{\infty}$-decay estimates. Meanwhile, he also showed that any weak solution with positive average blows up in finite time for any dimension space.

More recently [41], Wu et al. studied the problem (1.3) with $\gamma_{1}=\gamma_{2}=\gamma \in(0,1 / 2)$, in $1 \mathrm{D}$, and obtained the critical exponent

$$
F(p, q, \gamma):=\max \left\{1-\gamma+\frac{\gamma(p+1)}{p q-1}, 1-\gamma+\frac{\gamma(q+1)}{p q-1}\right\}-\frac{1}{2}
$$

They proved that if $F(p, q, \gamma)<0$ there exists a unique global small data solution of (1.3) and if $F(p, q, \gamma) \geq 0$ the non-existence of global solution can be derived with the initial data having positive average value.

Motivated by the papers [3],[22],[40],[41][4] in the present paper we consider the problem (1.1)(3.6 respectively), we will give conditions relating the space dimension $N$ with the system of parameters $\gamma_{1}$; $\gamma_{2} ; p_{1}, q_{1}, p_{2}$ and $q_{2}$ for which the solution of (1.1) (3.6 respectively) exists globally in time as well as $L^{\infty}$ decay estimates.

The best way to do this is to consider appropriately Lebesgue space where we can expect global wellposedness for this model, we observe that if $(u, v)$ is a solution for the system (1.1) with initial data $\left(u_{0}, v_{0}\right)$, then for all $\lambda>0,\left(u_{\lambda}, v_{\lambda}\right)=\left(\lambda^{k_{1}} u\left(\lambda^{2} t, \lambda x\right), \lambda^{k_{2}} u\left(\lambda^{2} t, \lambda x\right)\right)$ where

$$
k_{1}=\frac{\left(4-2 \gamma_{1}\right)\left(p_{2}-1\right)-\left(4-2 \gamma_{2}\right) q_{1}}{\left(\left(p_{1}-1\right)\left(p_{2}-1\right)-q_{1} q_{2}\right)}, k_{2}=\frac{\left(4-2 \gamma_{2}\right)\left(p_{1}-1\right)-\left(4-2 \gamma_{1}\right) q_{2}}{\left(\left(p_{1}-1\right)\left(p_{2}-1\right)-q_{1} q_{2}\right)},
$$

is also a solution of (1.1). If $\left(u_{0}, v_{0}\right) \in L^{r_{1}}\left(\mathbb{R}^{N}\right) \times L^{r_{2}}\left(\mathbb{R}^{N}\right)$, then the norms in $L^{r_{1}}\left(\mathbb{R}^{N}\right)$ and $L^{r_{2}}\left(\mathbb{R}^{N}\right)$ are preserved if and only if

$$
r_{1}=\frac{N\left(\left(p_{1}-1\right)\left(p_{2}-1\right)-q_{1} q_{2}\right)}{2\left[\left(2-\gamma_{1}\right)\left(p_{2}-1\right)-\left(2-\gamma_{2}\right) q_{1}\right]}, r_{2}=\frac{N\left(\left(p_{1}-1\right)\left(p_{2}-1\right)-q_{1} q_{2}\right)}{2\left[\left(2-\gamma_{2}\right)\left(p_{1}-1\right)-\left(2-\gamma_{1}\right) q_{2}\right]} .
$$

So we could expect that if $r_{1}>1$ and $r_{2}>1$ the mild solution of (1.1) with small initial data would exist globally. We will show in this paper that this result partially is not true. Using the diffusion phenomenon properties, we can obtain similar critical exponent results for the corresponding system of semilinear damped wave equations in low dimension space.

The rest of this paper is organized as follows. In the next section, we present some preliminary lemmas that we will need in the proof main results of this paper. We collect some basic facts and useful tools such as smoothing effect of the heat semigroup, $L^{p}-L^{q}$ estimates of the fundamental solutions of the damped wave equation. The local existence and the continuation results are presented in Section 3. Finally the proof of main results of this paper are proved in Sections 4 and 5.

In all this paper, $C$ will be a positive constant which may have different values at different places. Also, $L^{p}\left(\mathbb{R}^{N}\right)(1 \leq p \leq \infty)$ is the usual Lebesgue space with the norm $\|\cdot\|_{L^{p}\left(\mathbb{R}^{N}\right)}$. The space $C_{0}\left(\mathbb{R}^{N}\right)$ denotes the set of all continuous functions decaying to zero at infinity. For any $1 \leq p \leq \infty ; W^{1, p}\left(\mathbb{R}^{N}\right)$ denotes the usual Sobolev space

$$
W^{1, p}\left(\mathbb{R}^{N}\right):=\left\{f: \mathbb{R}^{N} \rightarrow \mathbb{R} ;\|f\|_{W^{1, p}\left(\mathbb{R}^{N}\right)}=\|f\|_{L^{p}\left(\mathbb{R}^{N}\right)}+\|\nabla f\|_{L^{p}\left(\mathbb{R}^{N}\right)}<+\infty\right\}
$$


For any Banach space $B$, we denote by $C([0, T] ; B)$ the space of continuous functions from $[0, T]$ into $B$ equipped with the uniform convergence $\sup _{t \in[0, T]}\|\cdot\|_{B}$, and $H^{l}\left(\mathbb{R}^{N}\right):=W^{2, l}\left(\mathbb{R}^{N}\right)(l \in \mathbb{N})$ stands for the usual Sobolev space equipped with the norm $\|f\|_{H^{l}\left(\mathbb{R}^{N}\right)}^{2}=\sum_{k=0}^{l}\left\|\partial_{x}^{k}\right\|_{L^{2}\left(\mathbb{R}^{N}\right)}^{2}<+\infty$.

\section{Preliminary lemmas}

Heat semigroup: Let us recall the definition of the so-called smoothing effect of the heat semi group on $\mathbb{R}^{N}$ and some related basic facts. For a complete presentation and more details, we refer the reader to [8].

Lemma 2.1. [8] Let $1 \leq r \leq s \leq \infty$. There exists a constant $C>0$ such that

$$
\left\|S(t) u_{0}\right\|_{L^{s}} \leq C t^{-\frac{N}{2}\left(\frac{1}{r}-\frac{1}{s}\right)}\left\|u_{0}\right\|_{L^{r}}, t>0
$$

for all $u_{0} \in L^{r}$. In particular for $u_{0} \in L^{r}\left(\mathbb{R}^{N}\right) \cap L^{s}\left(\mathbb{R}^{N}\right), 1 \leq r \leq s \leq \infty$, there exists $C=C(r, s)$ such that

$$
\left\|S(t) u_{0}\right\|_{L^{s}} \leq C(t+1)^{-\frac{N}{2}\left(\frac{1}{r}-\frac{1}{s}\right)}\left(\left\|u_{0}\right\|_{L^{r}}+\left\|u_{0}\right\|_{L^{s}}\right)
$$

for $t \geq 0$.

We will use also the following interpolation inequality

$$
\|u\|_{L^{s}} \leq\|u\|_{L^{s_{1}}}^{\theta}\|u\|_{L^{s_{2}}}^{1-\theta}
$$

for $u \in L^{s_{1}}\left(\mathbb{R}^{N}\right) \cap L^{s_{2}}\left(\mathbb{R}^{N}\right)$, where $s \in\left[s_{1}, s_{2}\right], \theta \in[0,1]$ with $\frac{1}{s}=\frac{\theta}{s_{1}}+\frac{1-\theta}{s_{2}}$.

We will need the following lemma which used in the proofs of Theorems 3.3 and 3.7.

Lemma 2.2. ([8]) Let $0 \leq a<1, b \geq 0$. Then there exists a constant $C>0$ depending only on $a$ and $b$ such that for all $t \geq 0$,

$$
\begin{aligned}
& \int_{0}^{t}(t-s)^{-a}(1+s)^{-b} d s \leq \begin{cases}C(1+t)^{-\min (a, b)} & \text { if } \max (a, b)>1, \\
C(1+t)^{-\min (a, b)} \ln (2+t) & \text { if } \max (a, b)=1, \\
C(1+t)^{1-a-b}, & \text { if } \max (a, b)<1 .\end{cases} \\
& \int_{0}^{t}(t-s+1)^{-a}(1+s)^{-b} d s \leq C(1+t)^{-b}, \text { for } t>0, a>1, a \geq b,
\end{aligned}
$$

and

$$
\int_{0}^{t} e^{-a(t-s)}(t-s)(1+s)^{-b} d s \leq C(1+t)^{-b}, a, b>0 .
$$




\section{Linear damped wave equation :}

Now, we recall some preliminary results concerning $L^{p}-L^{q}$ estimates of the fundamental solutions $K_{0}(t)$ and $K_{1}(t)$ to the linear damped wave equation

$$
\left\{\begin{array}{c}
u_{t t}-\Delta u+u_{t}=0,(t, x) \in(0,+\infty) \times \mathbb{R}^{N}, \\
u(0, x)=v_{0}(x), u_{t}(0, x)=v_{1}(x), x \in \mathbb{R}^{N} .
\end{array}\right.
$$

The solution $u(t)$ of linear equation (2.7) is given through the Fourier transform by $K_{0}(t)$ and $K_{1}(t)$ as

$$
u(t):=K_{0}(t) v_{0}+K_{1}(t)\left(\frac{1}{2} v_{0}+v_{1}\right) .
$$

Similarly, we introduce the evolution operators of the linear wave equation as follows:

$$
W_{0}(t) f:=\mathcal{F}^{-1}[\cos (t|\xi|) \hat{f}], W_{1}(t) g:=\mathcal{F}^{-1}\left[\frac{\sin (t|\xi|)}{|\xi|} \hat{g}\right] .
$$

In the following, we will consider the properties of these operators.

Lemma 2.3. ([23]) If $f \in L^{m}\left(\mathbb{R}^{N}\right) \cap H^{k+|\nu|-1}\left(\mathbb{R}^{N}\right)(1 \leq m \leq 2)$, then

$$
\left\|\partial_{t}^{k} \nabla_{x}^{\nu} K_{1}(t) * f\right\|_{2} \leq C(1+t)^{-N / 4-n /(2 m)-|\nu| / 2-k}\left(\|f\|_{m}+\|f\|_{H^{k+|\nu|-1}\left(\mathbb{R}^{N}\right)}\right) .
$$

Lemma 2.4. Let $1 \leq N \leq 3,1 \leq p \leq \infty, f \in W^{1, p}\left(\mathbb{R}^{N}\right)$ and $g \in L^{p}\left(\mathbb{R}^{N}\right)$. Then there exist some constants $C>0$ such that

$$
\begin{gathered}
\left\|W_{0}(t) f\right\|_{L^{p}\left(\mathbb{R}^{N}\right)} \leq C(1+|t|)\|f\|_{W^{1, p}\left(\mathbb{R}^{N}\right)}, t \neq 0, \\
\left\|W_{1}(t) g\right\|_{L^{p}\left(\mathbb{R}^{N}\right)} \leq C|t|\|g\|_{L^{p}\left(\mathbb{R}^{N}\right)}, t \neq 0,
\end{gathered}
$$

where $W_{0}(t) f$ and $W_{1}(t) g$ are defined by (2.8).

The proof of Lemma 2.4 is well known (cf. [27, 15]).

The following lemma will be used later and the proof of this lemma can be found in $[25,27,15,26,28])$, so we omit it here.

Lemma 2.5. Let $1 \leq N \leq 3,1 \leq q \leq p \leq \infty$ and $g \in L^{q}\left(\mathbb{R}^{N}\right)$. Then, there exist some constants $C>0$ such that for all $t>0$,

$$
\begin{aligned}
& \left\|\left(K_{0}(t)-e^{-\frac{t}{2}} W_{0}(t)-e^{-\frac{t}{2}} \frac{t}{8} W_{1}(t)\right) g\right\|_{L^{p}\left(\mathbb{R}^{N}\right)} \leq C(1+t)^{-\frac{N}{2}\left(\frac{1}{q}-\frac{1}{p}\right)}\|g\|_{L^{q}\left(\mathbb{R}^{N}\right)}, \\
& \left\|K_{1}(t) g-e^{-\frac{t}{2}} W_{1}(t) g\right\|_{L^{p}\left(\mathbb{R}^{N}\right)} \leq C(1+t)^{-\frac{N}{2}\left(\frac{1}{q}-\frac{1}{p}\right)}\|g\|_{L^{q}\left(\mathbb{R}^{N}\right)},
\end{aligned}
$$

where $K_{0}(t) g$ and $K_{1}(t) g$ are defined by (3.8)and (3.9).

\section{Main results}

In this section we state the main results of this paper. Before presenting the main theorems we introduce the de notion of mild solutions. 
Definition 3.1. For a mild solution of (1.1) we mean a function $(u, v) \in\left\{C\left([0, T) ; L^{1}\left(\mathbb{R}^{N}\right)\right)\right\}^{2} \cap$ $\left\{C\left(0, T ; C_{0}\left(\mathbb{R}^{N}\right)\right)\right\}^{2}$ satisfying the integral system

$$
\left\{\begin{array}{l}
u(t)=S(t) u_{0}+\int_{0}^{t} \int_{0}^{s}(s-\tau)^{-\gamma_{1}} S(t-s)|u(s)|^{p_{1}}|v(s)|^{q_{1}} d \tau d s, \\
v(t)=S(t) v_{0}+\int_{0}^{t} \int_{0}^{s}(s-\tau)^{-\gamma_{2}} S(t-s)|v(s)|^{p_{2}}|u(s)|^{q_{2}} d \tau d s,
\end{array}\right.
$$

where $\{S(t)\}_{t \geq 0}$ as the family of convolution operators with corresponding Gauss kernels $g(t, x)=$ $(4 \pi t)^{-N / 2} e^{-|x|^{2} /(4 t)}, t>0, x \in \mathbb{R}^{N}$, that is $S(t) f=g(t,) *$.$f , here *$ denotes the convolution product.

Our first result concerns the existence and uniqueness of mild solutions for the system (1.1).

Proposition 3.2 (Local existence of the heat system). Let $N \geq 1, p_{1}, q_{1}, p_{2}, q_{2} \geq 1, \gamma_{1}, \gamma_{2} \in[0,1)$ and $u_{0}, v_{0} \in C_{0}\left(\mathbb{R}^{N}\right)$. There exists a unique function $(u, v) \in\left\{C\left(\left(0, T_{\max }\right), C_{0}\left(\mathbb{R}^{N}\right)\right)\right\}^{2}$ solution of (1.1) such that either

(i) $T_{\max }=\infty$ (the solution is global) or else

(ii) $T_{\max }<\infty$ and $\lim _{t \rightarrow T_{\max }}\left(\|u(t)\|_{\infty}+\|v(t)\|_{\infty}\right)=\infty$ (the solution blows up in finite time).

Moreover if $\left(u_{0}, u_{1}\right) \in L^{r_{1}}\left(\mathbb{R}^{N}\right) \times L^{r_{2}}\left(\mathbb{R}^{N}\right)$ with $r_{1} \geq 1$ and $r_{2} \geq 1$, then $(u, v) \in C\left(\left(0, T_{\max }\right), L^{r_{1}}\left(\mathbb{R}^{N}\right)\right) \times$ $C\left(\left(0, T_{\max }\right), L^{r_{2}}\left(\mathbb{R}^{N}\right)\right)$ and

$$
\lim _{t \rightarrow T_{\max }}\left(\|u(t)\|_{L^{r_{1} \cap L^{\infty}}}+\|v(t)\|_{L^{r_{2} \cap L^{\infty}}}\right)=\infty
$$

when $T_{\max }<\infty$.

Under the above notations, our global existence result for the Cauchy problem (1.1) can be stated as in the following

Theorem 3.3 (Global existence of the heat system). Let $N$ be a positive integer. Let the real numbers $p_{1}, q_{1}, p_{2}, q_{2} \geq 1,0<\gamma_{1}, \gamma_{2}<1$ be such that

$$
\begin{aligned}
& {\left[\left(1-\gamma_{1}\right)\left(p_{2}-1\right)-\left(1-\gamma_{2}\right) q_{1}\right]\left(\left(p_{1}-1\right)\left(p_{2}-1\right)-q_{1} q_{2}\right)>0,} \\
& {\left[\left(1-\gamma_{2}\right)\left(p_{1}-1\right)-\left(1-\gamma_{1}\right) q_{2}\right]\left(\left(p_{1}-1\right)\left(p_{2}-1\right)-q_{1} q_{2}\right)>0,} \\
& {\left[p_{2}-q_{1}-1\right]\left(\left(p_{1}-1\right)\left(p_{2}-1\right)-q_{1} q_{2}\right)>0,} \\
& {\left[p_{1}-q_{2}-1\right]\left(\left(p_{1}-1\right)\left(p_{2}-1\right)-q_{1} q_{2}\right)>0 .}
\end{aligned}
$$

and $u_{0}, v_{0} \in C_{0}\left(\mathbb{R}^{N}\right)$. Let $(u, v) \in\left\{C\left(\left(0, T_{\max }\right), C_{0}\left(\mathbb{R}^{N}\right)\right)\right\}^{2}$.

Assume that

$$
\left\{\begin{array}{l}
\frac{N}{2}>1-\gamma_{1}+\frac{\left(2-\gamma_{1}\right)\left(p_{2}-1\right)-\left(2-\gamma_{2}\right) q_{1}}{\left(p_{1}-1\right)\left(p_{2}-1\right)-q_{1} q_{2}}, \\
\frac{N}{2}>1-\gamma_{2}+\frac{\left(2-\gamma_{2}\right)\left(p_{1}-1\right)-\left(2-\gamma_{1}\right) q_{2}}{\left(p_{1}-1\right)\left(p_{2}-1\right)-q_{1} q_{2}},
\end{array}\right.
$$




$$
\left\{\begin{array}{l}
\left(\left(p_{1}-1\right)\left(p_{2}-1\right)-q_{1} q_{2}\right) \times\left[p_{2}\left(\gamma_{1} p_{1}-1\right)-\gamma_{1} p_{1}+q_{1}\left(1-\gamma_{1} q_{2}\right)+1-\gamma_{2} q_{1}\right]>0 \\
\left(\left(p_{1}-1\right)\left(p_{2}-1\right)-q_{1} q_{2}\right) \times\left[p_{1}\left(\gamma_{2} p_{2}-1\right)-\gamma_{2} p_{2}+q_{2}\left(1-\gamma_{2} q_{1}\right)+1-\gamma_{1} q_{2}\right]>0
\end{array}\right.
$$

and

$$
\left\{\begin{array}{l}
\frac{N p_{1}}{2}\left[\frac{p_{1}}{r_{1}}+\frac{q_{1}}{r_{2}}\right]+\frac{N q_{1}}{2}\left[\frac{p_{2}}{r_{2}}+\frac{q_{2}}{r_{1}}\right]<2\left(p_{1}+q_{1}\right)+\frac{N}{2}, \\
\frac{N p_{2}}{2}\left[\frac{p_{2}}{r_{2}}+\frac{q_{2}}{r_{1}}\right]+\frac{N q_{2}}{2}\left[\frac{p_{1}}{r_{1}}+\frac{q_{1}}{r_{2}}\right]<2\left(p_{2}+q_{2}\right)+\frac{N}{2} .
\end{array}\right.
$$

Then there exists a constant $\varepsilon>0$ such that if the initial data satisfy $\left(u_{0}, v_{0}\right) \in L^{r_{1}}\left(\mathbb{R}^{N}\right) \times L^{r_{2}}\left(\mathbb{R}^{N}\right)$ and

$$
\left\|u_{0}\right\|_{\infty}+\left\|v_{0}\right\|_{\infty}+\left\|u_{0}\right\|_{r_{1}}+\left\|v_{0}\right\|_{r_{2}} \leq \varepsilon
$$

the problem (1.1) admits global solution $(u, v) \in C\left([0, \infty) ; L^{r_{1}}\left(\mathbb{R}^{N}\right) \times L^{r_{2}}\left(\mathbb{R}^{N}\right) \cap\left\{C_{0}\left(\mathbb{R}^{N}\right)\right\}^{2}\right)$ satisfies the following decay estimates

$$
\|u\|_{\infty} \leq C(t+1)^{-\alpha},\|v\|_{\infty} \leq C(t+1)^{-\beta}, \forall t \geq 0
$$

where $r_{1}, r_{2}$ given by (1.4) and

$$
\alpha=\frac{\left(1-\gamma_{1}\right)\left(p_{2}-1\right)-\left(1-\gamma_{2}\right) q_{1}}{\left(p_{1}-1\right)\left(p_{2}-1\right)-q_{1} q_{2}}, \beta=\frac{\left(1-\gamma_{2}\right)\left(p_{1}-1\right)-\left(1-\gamma_{1}\right) q_{2}}{\left(p_{1}-1\right)\left(p_{2}-1\right)-q_{1} q_{2}} .
$$

Similar consideration to the system for heat equations can be applied to the Cauchy

problem (1.1) for the system of damped wave equations in low dimensional space

$$
\left\{\begin{array}{l}
u_{t t}-\Delta u+u_{t}=\int_{0}^{t}(t-s)^{-\gamma_{1}}|u(s)|^{p_{1}}|v(s)|^{q_{1}} d s, t>0, x \in \mathbb{R}^{N}, \\
v_{t t}-\Delta v+v_{t}=\int_{0}^{t}(t-s)^{-\gamma_{2}}|v(s)|^{p_{2}}|u(s)|^{q_{2}} d s, t>0, x \in \mathbb{R}^{N}, \\
u(0, x)=u_{0}(x), u_{t}(0, x)=u_{1}(x), v(0, x)=v_{0}(x), v_{t}(0, x)=v_{1}(x), x \in \mathbb{R}^{N},
\end{array}\right.
$$

Let us give the definition of the solution for the Cauchy problem (3.6).

Definition 3.4. Let $u \in C\left([0, T) ; L^{1}\left(\mathbb{R}^{N}\right)\right) \cap L^{\infty}\left([0, T) ; L^{\infty}\left(\mathbb{R}^{N}\right)\right)$. Then the function $(u, v)$ is said to be a mild solution for the Cauchy problem (3.6) if there holds

$$
\left\{\begin{array}{l}
u(t)=K_{0}(t) u_{0}+K_{1}(t)\left(\frac{1}{2} u_{0}+u_{1}\right)+\int_{0}^{t} \int_{0}^{s}(s-\tau)^{-\gamma_{1}} K_{1}(t-s)|u(s)|^{p_{1}}|v(s)|^{q_{1}} d \tau d s, \\
v(t)=K_{0}(t) v_{0}+K_{1}(t)\left(\frac{1}{2} v_{0}+v_{1}\right)+\int_{0}^{s} \int_{0}^{s}(s-\tau)^{-\gamma_{2}} K_{1}(t-s)|v(s)|^{p_{2}}|u(s)|^{q_{2}} d \tau d s,
\end{array}\right.
$$

for all $(x, t) \in \mathbb{R}^{N} \times[0, T)$, where the evolution operators $K_{0}(t)$ and $K_{1}(t)$ solutions of the linear damped wave equation are given by

$$
\left(K_{0}(t) \phi\right)(x):=\mathcal{F}^{-1}\left[e^{-\frac{t}{2}} \cos \left(t \sqrt{|\xi|^{2}-\frac{1}{4}}\right) \mathcal{F}[\phi]\right](x),
$$




$$
\left(K_{1}(t) \phi\right)(x):=\mathcal{F}^{-1}\left[e^{-\frac{t}{2}} \frac{\sin \left(t \sqrt{|\xi|^{2}-\frac{1}{4}}\right)}{\sqrt{|\xi|^{2}-\frac{1}{4}}} \mathcal{F}[\phi]\right](x) .
$$

Here we denote the Fourier and Fourier inverse transform by $\mathcal{F}$ and $\mathcal{F}^{-1}$, respectively. In particular, when $N=1,2,3$, like in the paper [28], (3.7) can also be written as follows:

$$
\left\{\begin{array}{l}
u(t, .)=K_{1}(t)\left(\frac{1}{2} u_{0}+u_{1}\right)+K_{0}(t) u_{0}+\int_{0}^{t}\left(K_{1}(t-s)-e^{-\frac{t-s}{2}} W_{1}(t-s)\right) \\
\times \int_{0}^{s}(s-\tau)^{-\gamma_{1}}|u(\tau, .)|^{p_{1}}|v(\tau, .)|^{q_{1}} d \tau d s+\int_{0}^{t} e^{-\frac{t-s}{2}} W_{1}(t-s) \int_{0}^{s}(s-\tau)^{-\gamma_{1}}|u(\tau, .)|^{p_{1}}|v(\tau, .)|^{q_{1}} d \tau d s, \\
v(t, .)=K_{1}(t)\left(\frac{1}{2} v_{0}+v_{1}\right)+K_{0}(t) v_{0}+\int_{0}^{t}\left(K_{1}(t-s)-e^{-\frac{t-s}{2}} W_{1}(t-s)\right) \\
\times \int_{0}^{s}(s-\tau)^{-\gamma_{2}}|v(\tau, .)|^{p_{2}}|u(\tau, .)|^{q_{2}} d \tau d s+\int_{0}^{t} e^{-\frac{t-s}{2}} W_{1}(t-s) \int_{0}^{s}(s-\tau)^{-\gamma_{2}}|v(\tau, .)|^{p_{2}}|u(\tau, .)|^{q_{2}} d \tau d s .
\end{array}\right.
$$

Proposition 3.5 (Local existence for the damped wave system). Let $1 \leq N \leq 3, p_{1}, q_{1}, p_{2}, q_{2} \geq$ $1, \gamma_{1}, \gamma_{2} \in[0,1)$ and $\left(u_{0}, u_{1}\right),\left(v_{0}, v_{1}\right) \in W^{1, \infty}\left(\mathbb{R}^{N}\right) \times \cap L^{\infty}\left(\mathbb{R}^{N}\right)$. There exists a unique function $(u, v) \in\left\{C\left(\left(0, T_{\max }\right), L^{\infty}\left(\mathbb{R}^{N}\right)\right)\right\}^{2}$ solution of (3.6) such that either

(i) $T_{\max }=\infty$ (the solution is global) or else

(ii) $T_{\max }<\infty$ and $\lim _{t \rightarrow T_{\max }}\left(\|u(t)\|_{\infty}+\|v(t)\|_{\infty}\right)=\infty$ (the solution blows up in finite time).

Moreover if $\left(u_{0}, u_{1}\right) \in W^{1,1}\left(\mathbb{R}^{N}\right) \times L^{1}\left(\mathbb{R}^{N}\right)$ and $\left(v_{0}, v_{1}\right) \in W^{1,1}\left(\mathbb{R}^{N}\right) \times L^{1}\left(\mathbb{R}^{N}\right)$, then $(u, v) \in$ $C\left(\left[0, T_{\max }\right), L^{r_{1}}\left(\mathbb{R}^{N}\right) \times L^{r_{2}}\left(\mathbb{R}^{N}\right)\right)$ for any $r_{1} \geq 1$ and $r_{2} \geq 1$, and $\lim _{t \rightarrow T_{\max }}\left(\|u(t)\|_{L^{r_{1} \cap L^{\infty}}}+\right.$ $\left.\|v(t)\|_{L^{r_{2} \cap L^{\infty}}}\right)=\infty$, when $T_{\max }<\infty$.

Furthermore the solution $u, v \in C\left(\left[0, T_{\max }\right), H^{1}\left(\mathbb{R}^{N}\right)\right) \cap C^{1}\left(\left[0, T_{\max }\right), L^{2}\left(\mathbb{R}^{N}\right)\right)$.

Remark 3.6. The proof of Proposition 3.5 is omitted here since it follows by combining the proofs of Propositions 3.2 and 3.6 in [4] with Lemmas 3.5, 2.3, 2.5 togethers.

The main purpose of our next theorem is to show that the same result holds also for the Cauchy problem (3.6)

Theorem 3.7 (Global existence). Let $1 \leq N \leq 3$ be a positive integer. Let the real numbers $p_{1}, q_{1}, p_{2}, q_{2} \geq$ $1,0<\gamma_{1}, \gamma_{2}<1$ be such that

$$
\begin{gathered}
{\left[\left(1-\gamma_{1}\right)\left(p_{2}-1\right)-\left(1-\gamma_{2}\right) q_{1}\right]\left(\left(p_{1}-1\right)\left(p_{2}-1\right)-q_{1} q_{2}\right)>0} \\
{\left[\left(1-\gamma_{2}\right)\left(p_{1}-1\right)-\left(1-\gamma_{1}\right) q_{2}\right]\left(\left(p_{1}-1\right)\left(p_{2}-1\right)-q_{1} q_{2}\right)>0,} \\
{\left[p_{2}-q_{1}-1\right]\left(\left(p_{1}-1\right)\left(p_{2}-1\right)-q_{1} q_{2}\right)>0,} \\
{\left[p_{1}-q_{2}-1\right]\left(\left(p_{1}-1\right)\left(p_{2}-1\right)-q_{1} q_{2}\right)>0 .}
\end{gathered}
$$


Assume that

$$
\begin{aligned}
& \left\{\begin{array}{l}
\frac{N}{2}>1-\gamma_{1}+\frac{\left(2-\gamma_{1}\right)\left(p_{2}-1\right)-\left(2-\gamma_{2}\right) q_{1}}{\left(p_{1}-1\right)\left(p_{2}-1\right)-q_{1} q_{2}}, \\
\frac{N}{2}>1-\gamma_{2}+\frac{\left(2-\gamma_{2}\right)\left(p_{1}-1\right)-\left(2-\gamma_{1}\right) q_{2}}{\left(p_{1}-1\right)\left(p_{2}-1\right)-q_{1} q_{2}},
\end{array}\right. \\
& \left\{\begin{array}{l}
\left(\left(p_{1}-1\right)\left(p_{2}-1\right)-q_{1} q_{2}\right) \times\left[p_{2}\left(\gamma_{1} p_{1}-1\right)-\gamma_{1} p_{1}+q_{1}\left(1-\gamma_{1} q_{2}\right)+1-\gamma_{2} q_{1}\right]>0, \\
\left(\left(p_{1}-1\right)\left(p_{2}-1\right)-q_{1} q_{2}\right) \times\left[p_{1}\left(\gamma_{2} p_{2}-1\right)-\gamma_{2} p_{2}+q_{2}\left(1-\gamma_{2} q_{1}\right)+1-\gamma_{1} q_{2}\right]>0,
\end{array}\right.
\end{aligned}
$$

and

$$
\left\{\begin{array}{l}
\frac{N p_{1}}{2}\left[\frac{p_{1}}{r_{1}}+\frac{q_{1}}{r_{2}}\right]+\frac{N q_{1}}{2}\left[\frac{p_{2}}{r_{2}}+\frac{q_{2}}{r_{1}}\right]<2\left(p_{1}+q_{1}\right)+\frac{N}{2}, \\
\frac{N p_{2}}{2}\left[\frac{p_{2}}{r_{2}}+\frac{q_{2}}{r_{1}}\right]+\frac{N q_{2}}{2}\left[\frac{p_{1}}{r_{1}}+\frac{q_{1}}{r_{2}}\right]<2\left(p_{2}+q_{2}\right)+\frac{N}{2} .
\end{array}\right.
$$

Then there exists a positive constant $\varepsilon>0$ such that if the initial data satisfy $\left(u_{i}, v_{i}\right) \in\left\{W^{1-i, 1}\left(\mathbb{R}^{N}\right)\right.$ $\left.\times W^{1-i, \infty}\left(\mathbb{R}^{N}\right)\right\}^{2}, i=0,1$, and

$$
\left\|u_{0}\right\|_{W^{1,1} \cap W^{1, \infty}}+\left\|v_{0}\right\|_{W^{1,1} \cap W^{1, \infty}}+\left\|u_{1}\right\|_{L^{1} \cap L^{\infty}}+\left\|v_{1}\right\|_{L^{1} \cap L^{\infty}} \leq \varepsilon,
$$

the corresponding problem (3.6) admits global solution

$$
\begin{aligned}
(u, v) \in & C\left([0, \infty) ; L^{r_{1}}\left(\mathbb{R}^{N}\right) \cap L^{\infty}\left(\mathbb{R}^{N}\right) \cap H^{1}\left(\mathbb{R}^{N}\right)\right) \cap C^{1}\left([0, \infty) ; L^{2}\left(\mathbb{R}^{N}\right)\right) \\
& \times C\left([0, \infty) ; L^{r_{2}}\left(\mathbb{R}^{N}\right) \cap L^{\infty}\left(\mathbb{R}^{N}\right) \cap H^{1}\left(\mathbb{R}^{N}\right)\right) \cap C^{1}\left([0, \infty) ; L^{2}\left(\mathbb{R}^{N}\right)\right),
\end{aligned}
$$

satisfies the following decay estimates

$$
\|u\|_{\infty} \leq C(t+1)^{-\alpha}, \quad\|v\|_{\infty} \leq C(t+1)^{-\beta}, \forall t \geq 0
$$

where $r_{1}, r_{2}$ given by (1.4) and

$$
\alpha=\frac{\left(1-\gamma_{1}\right)\left(p_{2}-1\right)-\left(1-\gamma_{2}\right) q_{1}}{\left(p_{1}-1\right)\left(p_{2}-1\right)-q_{1} q_{2}}, \beta=\frac{\left(1-\gamma_{2}\right)\left(p_{1}-1\right)-\left(1-\gamma_{1}\right) q_{2}}{\left(p_{1}-1\right)\left(p_{2}-1\right)-q_{1} q_{2}} .
$$

Remarks 3.8. 1) From the definition of $r_{1}$ and $r_{1}$, we note that the left hand sides of inequalities (3.5) and (3.13) are independent of the dimension $N$.

2) Notice that the above results remain true for $p_{1}, p_{2} \geq 0, q_{1}, q_{2} \geq 1$ with $p_{1}+q_{1}>1$ and $p_{2}+q_{2}>1$.

3) Theorem 3.3 and 3.7 are sharp in the case (3.5) (respectively, (3.13)), see (4)-(14) and generalizes Theorem 1.1 of [3]. In fact let $p_{1}=p_{2}=0, q_{1}=q_{2}=q$ and $\gamma_{1}=\gamma_{2}=\gamma$. Conditions (3.3) and (3.4) reduce to $q\left(\frac{N}{2}+\gamma-1\right) \geq \frac{N}{2}+1$ or $q \gamma \geq 1$ respectively. From these facts, it is possible to conclude that, if (3.4) and (3.13) are valid, then the value of the Fujita critical exponent is $p_{*}$.

4) When $p_{1}=p_{2}=0$. These results are in agreement with results obtained by [22, Theorem 2] and [4, Theorem 2.1, 2.2]. In particular the condition (3.13) of Theorem 3.7 improves the one in [4, Formula (10)]

5) The same result can be stated for more general nonlinearities, namely for $f_{i}, i=1,2$

$$
\begin{aligned}
& \left|f_{1}(u, v)-f_{1}(\bar{u}, v)\right| \leq C|u-\bar{u}|\left(|u|^{p_{1}-1}+|\bar{u}|^{p_{1}-1}\right)|v|^{q_{1}}, \\
& \left|f_{1}(u, v)-f_{1}(u, \bar{v})\right| \leq C|v-\bar{v}|\left(|v|^{q_{1}-1}+|\bar{v}|^{q_{1}-1}\right)|u|^{p_{1}}, \\
& \left|f_{2}(u, v)-f_{2}(\bar{u}, v)\right| \leq C|u-\bar{u}|\left(|u|^{q_{2}-1}+|\bar{u}|^{q_{2}-1}\right)|v|^{p_{2}}, \\
& \left|f_{2}(u, v)-f_{2}(u, \bar{v})\right| \leq C|v-\bar{v}|\left(|v|^{p_{2}-1}+|\bar{v}|^{p_{2}-1}\right)|u|^{q_{2}} .
\end{aligned}
$$


Proof of Proposition 3.2. The proof relies in the Banach fixed point theorem. Given $M>0$ such that let

$$
K:=\left\{(u, v) \in\left\{L^{\infty}\left([0, T), C_{0}\left(\mathbb{R}^{N}\right)\right)\right\}^{2} ;\|u(t)\|_{\infty} \leq M+1,\|v(t)\|_{\infty} \leq M+1\right\},
$$

where $T>0$ will be chosen later. The space $K$ equipped with the metric

$$
d((u, v),(\bar{u}, \bar{v}))=\sup _{t \in(0, T)}\|u(t)-\bar{u}(t)\|_{\infty}+\sup _{t \in(0, T)}\|v(t)-\bar{v}(t)\|_{\infty},
$$

is a complete metric space. Define the mapping $\Phi: K \times K \rightarrow\left\{L^{\infty}\left([0, T), C_{0}\left(\mathbb{R}^{N}\right)\right)\right\}^{2}$ as the following

$$
\Phi(u, v)=\left(\Phi_{1}(u, v), \Phi_{2}(u, v)\right),(u, v) \in K
$$

where

$$
\left\{\begin{array}{l}
\Phi_{1}(u, v)=S(t) u_{0}+\int_{0}^{t} \int_{0}^{s} S(t-s)(s-\tau)^{-\gamma_{1}}|u(\tau)|^{p_{1}}|v(\tau)|^{q_{1}} d \tau d s, \\
\Phi_{2}(u, v)=S(t) v_{0}+\int_{0}^{t} \int_{0}^{s} S(t-s)(s-\tau)^{-\gamma_{2}}|v(\tau)|^{p_{2}}|u(\tau)|^{q_{2}} d \tau d s .
\end{array}\right.
$$

First,we claim that $\Phi$ maps $K$ it into itself, and is contraction when $T$ is chosen appropriately. We have for $(u, v) \in K$ that

$$
\begin{aligned}
\left\|\Phi_{1}(u, v)\right\|_{\infty} & \leq\left\|u_{0}\right\|_{\infty}+\int_{0}^{t} \int_{0}^{s}(s-\tau)^{-\gamma_{1}}\|u\|_{\infty}^{p_{1}}\|v\|_{\infty}^{q_{1}} d \tau d s \\
& \leq\left\|u_{0}\right\|_{\infty}+T^{2-\gamma_{1}}(M+1)^{p_{1}+q_{1}} .
\end{aligned}
$$

Similarly

$$
\begin{aligned}
\left\|\Phi_{2}(u, v)\right\|_{\infty} & \leq\left\|u_{0}\right\|_{\infty}+\int_{0}^{t} \int_{0}^{s}(s-\tau)^{-\gamma_{2}}\|v\|_{\infty}^{p_{2}}\|u\|_{\infty}^{q_{2}} d \tau d s \\
& \leq\left\|v_{0}\right\|_{\infty}+T^{2-\gamma_{2}}(M+1)^{p_{2}+q_{2}}
\end{aligned}
$$

We will prove that for $T>0$ sufficiently small, $\Phi$ is a contraction map from $K$ into itself. We consider $\left(u_{1}, u_{2}\right),\left(v_{1}, v_{2}\right) \in K$. For any $t \in[0, T)$, we have

$$
\begin{aligned}
\left\|\Phi_{1}\left(u_{1}, u_{2}\right)-\Phi_{1}\left(v_{1}, v_{2}\right)\right\|_{\infty} \leq & \int_{0}^{t} \int_{0}^{s}|| S(t-s)(s-\tau)^{-\gamma_{1}}\left(\left|u_{1}\right|^{p_{1}}\left|u_{2}\right|^{q_{1}}-\left|v_{1}\right|^{p_{1}}\left|u_{2}\right|^{q_{1}}\right. \\
& \left.+\left|v_{1}\right|^{p_{1}}\left|u_{2}\right|^{q_{1}}-\left|v_{1}\right|^{p_{1}}\left|v_{2}\right|^{q_{1}}\right) \|_{\infty} d \tau d s .
\end{aligned}
$$

Then, thanks to Lemma 2.1, we get

$$
\begin{aligned}
\left\|\Phi_{1}\left(u_{1}, u_{2}\right)-\Phi_{1}\left(v_{1}, v_{2}\right)\right\|_{\infty} \leq & C \int_{0}^{t} \int_{0}^{s}(s-\tau)^{-\gamma_{1}}\left\|u_{2}\right\|_{\infty}^{q_{1}}\left(\left\|u_{1}\right\|_{\infty}^{p_{1}-1}+\left\|v_{1}\right\|_{\infty}^{p_{1}-1}\right)\left\|u_{1}-v_{1}\right\|_{\infty} \\
& +\left\|v_{1}\right\|_{\infty}^{p_{1}}\left(\left\|u_{2}\right\|_{\infty}^{q_{1}-1}+\left\|v_{2}\right\|_{\infty}^{q_{1}-1}\right)\left\|u_{2}-v_{2}\right\|_{\infty} d \tau d s .
\end{aligned}
$$


Therefore

$$
\left\|\Phi_{1}\left(u_{1}, u_{2}\right)-\Phi_{1}\left(v_{1}, v_{2}\right)\right\|_{\infty} \leq 2 C T^{2-\gamma_{1}}(M+1)^{p_{1}+q_{1}-1}\left(\sup _{t \in(0, T)}\left\|u_{1}-v_{1}\right\|+\sup _{t \in(0, T)}\left\|u_{2}-v_{2}\right\|_{\infty}\right) .
$$

Similarly, we have

$$
\left\|\Phi_{2}\left(u_{1}, u_{2}\right)-\Phi_{2}\left(v_{1}, v_{2}\right)\right\|_{\infty} \leq 2 C T^{2-\gamma_{2}}(M+1)^{p_{2}+q_{2}-1}\left(\sup _{t \in(0, T)}\left\|u_{1}-v_{1}\right\|_{\infty}+\sup _{t \in(0, T)}\left\|u_{2}-v_{2}\right\|_{\infty}\right),
$$

for some positive constant $C>0$. From (3.14)-(3.17) it follows that if $T$ sufficiently small, then $\Phi$ is a strict contraction from $K$ into itself, so $\Phi$ has a unique fixed point $(u, v)$ in $K$, which is a solution of (1.1).

It is easy to prove that for each $T^{\prime}>0$ system (3.15) has at most one solution which lies to $K\left(T^{\prime}\right)$. In fact, let $\left(u_{1}, v_{1}\right),\left(u_{2}, v_{2}\right) \in K\left(T^{\prime}\right)$ be two solutions of system (3.1); then

$$
\begin{aligned}
u_{1}(t, .)-u_{2}(t, .)= & \int_{0}^{t} S(t-s) \int_{0}^{s}(s-\tau)^{-\gamma_{1}}\left(\left(\left|u_{1}(\tau)\right|^{p_{1}}-\left|u_{2}(\tau)\right|^{p_{1}}\right)\left|v_{1}(\tau)\right|^{q_{1}}\right. \\
& \left.+\left(\left|v_{1}(\tau)\right|^{q_{1}}-\left|v_{2}(\tau)\right|^{q_{1}}\right)\left|u_{2}(\tau)\right|^{p_{1}}\right) d \sigma d s \\
v_{1}(t, .)-v_{2}(t, .)= & \int_{0}^{t} S(t-s) \int_{0}^{s}(s-\tau)^{-\gamma_{2}}\left(\left(\left|v_{1}(\tau)\right|^{p_{2}}-\left|v_{2}(\tau)\right|^{p_{2}}\right)\left|u_{1}(\tau)\right|^{q_{2}}\right. \\
& \left.+\left|v_{2}(\tau)\right|^{p_{2}}\left(\left|u_{1}(\tau)\right|^{q_{2}}-\left|u_{2}(\tau)\right|^{q_{2}}\right)\right) d \sigma d s
\end{aligned}
$$

By the help of the following inequality

$$
\left.|| u_{1}\right|^{k}-\left|u_{2}\right|^{k}|\leq C| u_{1}-u_{2} \mid\left(\left|u_{1}\right|^{k-1}+\left|u_{2}\right|^{k-1}\right)
$$

for every $u_{1}, u_{2} \in \mathbb{R}$ and all $k \geq 1$ and by the definition of $K\left(T^{\prime}\right)$. Thus from (3.17), (3.18), Lemma 2.1 , we get

$$
\begin{aligned}
& \sup _{t \in\left[0, T^{\prime}\right]}\left\|u_{1}(t, .)-u_{2}(t, .)\right\|_{\infty}+\sup _{t \in\left[0, T^{\prime}\right]}\left\|v_{1}(t, .)-v_{2}(t, .)\right\|_{\infty} \leq \\
& C\left(T^{\prime}\right) \int_{0}^{t}\left(\sup _{\tau \in[0, s]}\left\|u_{1}(\tau, .)-u_{2}(\tau, .)\right\|_{\infty}+\sup _{t \in[0, s]}\left\|v_{1}(\tau, .)-v_{2}(\tau, .)\right\|_{\infty}\right) d s,
\end{aligned}
$$

where $C\left(T^{\prime}\right)$ constant dependent on $T^{\prime}$. From (3.20) and Gronwall's inequality, we find $u_{1}=u_{2}$ and $v_{1}=v_{2}$, i.e. system (3.15) has at most one solution which belongs to $K\left(T^{\prime}\right)$. Due to uniqueness, it follows that the solution $(u, v)$ can be extended to a maximal interval $\left[0, T_{\max }\right)$. Note that if $0 \leq t \leq$ $t+\tau \leq T_{\max }$, we have 


$$
\begin{aligned}
& u(t+\tau)=S(\tau) u(t)+\int_{0}^{\tau} S(\tau-s) \int_{0}^{\tau}(\tau-\sigma)^{-\gamma_{1}}|u(t+\sigma)|^{p_{1}}|v(t+\sigma)|^{q_{1}} d \sigma d s \\
& \quad+\int_{0}^{\tau} S(\tau-s) \int_{0}^{\tau}(t+s-\sigma)^{-\gamma_{1}}|u(\sigma)|^{p_{1}}|v(\sigma)|^{q_{1}} d \sigma d s, \\
& v(t+\tau)=S(\tau) v(t)+\int_{0}^{\tau} S(\tau-s) \int_{0}^{\tau}(\tau-\sigma)^{-\gamma_{2}}|v(t+\sigma)|^{p_{2}}|u(t+\sigma)|^{q_{2}} d \sigma d s \\
& \quad+\int_{0}^{\tau} S(\tau-s) \int_{0}^{\tau}(t+s-\sigma)^{-\gamma_{2}}|v(\sigma)|^{p_{2}}|u(\sigma)|^{q_{2}} d \sigma d s .
\end{aligned}
$$

By the fixed point argument, it follows from (3.21) that if $\|u(t)\|_{L^{\infty}\left((0, T) \times \mathbb{R}^{N}\right)}+\|v(t)\|_{L^{\infty}\left((0, T) \times \mathbb{R}^{N}\right)}<$ $\infty$, then $(u, v)$ can be extended to interval $\left[0, T^{\prime}\right)$ with $T^{\prime}>T$. This shows that if $T_{\max }<\infty$, then $\lim _{t \rightarrow T_{\max }}\|u(t)\|_{\infty}+\|v(t)\|_{\infty}=\infty$.

To show the remaining part of Theorem 3.2, we use again a fixed point argument. Consider the space

$$
E=L^{\infty}\left([0, T), L^{r_{1}}\left(\mathbb{R}^{N}\right) \times L^{r_{2}}\left(\mathbb{R}^{N}\right) \cap\left\{C_{0}\left(\mathbb{R}^{N}\right)\right\}^{2}\right)
$$

and

$$
K=\left\{\bar{u}=(u, v) \in E ;\|u(t)\|_{\infty},\|v(t)\|_{\infty},\|u(t)\|_{r_{1}},\|v(t)\|_{r_{2}}<M+1, \text { for all } t \in(0, T)\right\},
$$

where $M \geq \max \left\{\left\|u_{0}\right\|_{\infty},\left\|v_{0}\right\|_{\infty},\left\|u_{0}\right\|_{r_{1}},\left\|v_{0}\right\|_{r_{2}}\right\}$. The space $(K, d)$ with the metric

$$
d(\bar{u}, \bar{v})=\max _{i=1,2}\left\{\sup _{t \in(0, T)}\left\|u_{i}(t)-v_{i}(t)\right\|_{\infty}, \sup _{t \in(0, T)}\left\|u_{i}(t)-v_{i}(t)\right\|_{r_{i}}\right\}
$$

where $\bar{u}=\left(u_{1}, u_{2}\right), \bar{v}=\left(v_{1}, v_{2}\right)$ is a complete metric space.

Since $r_{1} \geq 1$ and $r_{2} \geq 1$, we can choose $\xi, \omega, \xi_{1}, \omega_{1} \geq 1$ by taking $\frac{1}{\xi}=\frac{1-k}{r_{1}}, \frac{1}{\omega}=\frac{1-k}{r_{2}}, \frac{1}{\xi_{1}}=\frac{1-k_{1}}{r_{1}}$ and $\frac{1}{\omega_{2}}=\frac{1-k}{r_{2}}$ for some constants $0<k, k_{1}<1$ satisfying

$$
\begin{aligned}
& \frac{p_{1}}{\xi}+\frac{q_{1}}{\omega} \leq 1,0 \leq \frac{N}{2}\left(\frac{p_{1}}{\xi}+\frac{q_{1}}{\omega}-\frac{1}{r_{1}}\right) \leq 1, \frac{1}{\xi} \leq \frac{1}{r_{1}}, \frac{1}{\omega} \leq \frac{1}{r_{2}}, \\
& \frac{p_{2}}{\omega_{1}}+\frac{q_{2}}{\xi_{1}} \leq 1,0 \leq \frac{N}{2}\left(\frac{p_{2}}{\omega_{1}}+\frac{q_{2}}{\xi_{1}}-\frac{1}{r_{2}}\right) \leq 1, \frac{1}{\omega_{1}} \leq \frac{1}{r_{2}}, \frac{1}{\xi_{1}} \leq \frac{1}{r_{1}}
\end{aligned}
$$

Using the smoothing effect of the heat semigroup (2.1), interpolation inequality (2.3) and (3.22), we find

$$
\begin{aligned}
\left\|\Phi_{1}(u, v)\right\|_{r_{1}} & =\left\|u_{0}\right\|_{r_{1}}+\int_{0}^{t}\left\|S(t-s) \int_{0}^{s}(s-\tau)^{-\gamma_{1}}|u(\tau)|^{p_{1}}|v(\tau)|^{q_{1}}\right\|_{r_{1}} d \tau d s \\
& \leq\left\|u_{0}\right\|_{r_{1}}+\int_{0}^{t} \int_{0}^{s}(t-s)^{-\frac{N}{2}\left(\frac{p_{1}}{\xi}+\frac{q_{1}}{\omega}-\frac{1}{r_{1}}\right)}(s-\tau)^{-\gamma_{1}}\|u\|_{\xi}^{p_{1}}\|v\|_{\omega}^{q_{1}} d \tau d s
\end{aligned}
$$




$$
\begin{aligned}
& \leq\left\|u_{0}\right\|_{r_{1}}+\int_{0}^{t} \int_{0}^{s}(t-s)^{-\frac{N}{2}\left(\frac{p_{1}}{\xi}+\frac{q_{1}}{\omega}-\frac{1}{r_{1}}\right)}(s-\tau)^{-\gamma_{1}}\|u\|_{r_{1}}^{\frac{r_{1}}{\xi} p_{1}}\|u\|_{\infty}^{\left(1-\frac{r_{1}}{\xi}\right) p_{1}} \\
& \times\|v\|_{r_{2}}^{\frac{r_{2}}{\omega}} q_{1}\|v\|_{\infty}^{\left(1-\frac{r_{2}}{\omega}\right) q_{1}} d \tau d s \\
& \leq\left\|u_{0}\right\|_{r_{1}}+\int_{0}^{t} \int_{0}^{s}(t-s)^{-\frac{N}{2}\left(\frac{p_{1}}{\xi}+\frac{q_{1}}{\omega}-\frac{1}{r_{1}}\right)}(s-\tau)^{-\gamma_{1}}\|u\|_{r_{1}}^{\frac{r_{1}}{\xi} p_{1}}\|u\|_{\infty}^{\left(1-\frac{r_{1}}{\xi}\right) p_{1}} \\
& \times\|v\|_{r_{2}}^{\frac{r_{2}}{\omega}} q_{1}\|v\|_{\infty}^{\left(1-\frac{r_{2}}{\omega}\right) q_{1}} d \tau d s \\
& \leq\left\|u_{0}\right\|_{r_{1}}+T^{2-\gamma_{1}-\frac{N}{2}\left(\frac{p_{1}}{\xi}+\frac{q_{1}}{\omega}-\frac{1}{r_{1}}\right)}(M+1)^{p_{1}+q_{1}}
\end{aligned}
$$

Thus

$$
\left\|\Phi_{1}(u, v)\right\|_{r_{1}} \leq M+1
$$

if $T$ is small enough. Analogously, taking $T$ eventually smaller, we get

$$
\left\|\Phi_{2}(u, v)\right\|_{r_{2}} \leq\left\|v_{0}\right\|_{r_{2}}+T^{2-\gamma_{2}-\frac{N}{2}\left(\frac{p_{2}}{\omega_{1}}+\frac{q_{2}}{\xi_{1}}-\frac{1}{r_{2}}\right)}(M+1)^{p_{2}+q_{2}} \leq M+1 .
$$

Taking a smaller $T$ if necessary, show that $\Phi$ is a contraction in $K$, indeed

$$
\begin{aligned}
\left|\Phi_{1}\left(u_{1}, u_{2}\right)-\Phi_{1}\left(v_{1}, v_{2}\right)\right| \leq & \int_{0}^{t} \int_{0}^{s}\left|S(t-s)(s-\tau)^{-\gamma_{1}}\left(\left|u_{1}\right|^{p_{1}}\left|u_{2}\right|^{q_{1}}-\left|v_{1}\right|^{p_{1}}\left|v_{2}\right|^{q_{1}}\right)\right| d \tau d s \\
\leq & \int_{0}^{t} \int_{0}^{s} S(t-s)(s-\tau)^{-\gamma_{1}} \times\left.|| u_{1}\right|^{p_{1}}\left[\left|u_{2}\right|^{q_{1}}-\left|v_{2}\right|^{q_{1}}\right] \mid d \tau d s \\
& +\left.\int_{0}^{t} \int_{0}^{s} S(t-s)(s-\tau)^{-\gamma_{1}}\left|v_{2}\right|^{q_{1}}|| u_{1}\right|^{p_{1}}-\left|v_{1}\right|^{p_{1}} \mid d \tau d s
\end{aligned}
$$

Therefore, from (2.1) and the Hölder inequality, we have

$$
\begin{aligned}
\left\|\Phi_{1}\left(u_{1}, u_{2}\right)-\Phi_{1}\left(v_{1}, v_{2}\right)\right\|_{r_{1}} \leq & \int_{0}^{t} \int_{0}^{s}(t-s)^{-\frac{N}{2}\left(\frac{p_{1}}{\xi}+\frac{q_{1}}{\omega}-\frac{1}{r_{1}}\right)}(s-\tau)^{-\gamma_{1}} \\
& \times\left[\left\|u_{1}\right\|_{\eta}^{p_{1}}\left(\left\|u_{2}\right\|_{\omega}^{q_{1}-1}+\left\|v_{2}\right\|_{\omega}^{q_{1}-1}\right)\left\|u_{2}-v_{2}\right\|_{\omega}\right. \\
& \left.+\left\|v_{2}\right\|_{\omega}^{q_{1}}\left(\left\|u_{1}\right\|_{\xi}^{p_{1}-1}+\left\|v_{1}\right\|_{\xi}^{p_{1}-1}\right)\left\|u_{1}-v_{1}\right\|_{\xi}\right] d \tau d s
\end{aligned}
$$

Hence

$$
\left\|\Phi_{1}(\bar{u})-\Phi_{1}(\bar{v})\right\|_{r_{1}} \leq 2(M+1)^{p_{1}+q_{1}-1} \int_{0}^{t} \int_{0}^{s}(t-s)^{-\frac{N}{2}\left(\frac{p_{1}}{\xi}+\frac{q_{1}}{\omega}-\frac{1}{r_{1}}\right)}(s-\tau)^{-\gamma_{1}} d \tau d s d(\bar{u}, \bar{v}) .
$$


That is

$$
\left\|\Phi_{1}(\bar{u})-\Phi_{1}(\bar{v})\right\|_{r_{1}} \leq C T^{2-\gamma_{1}-\frac{N}{2}\left(\frac{q_{1}}{\omega}+\frac{p_{1}}{\eta}-\frac{1}{r_{1}}\right)}(M+1)^{p_{1}+q_{1}-1} d(\bar{u}, \bar{v})
$$

By analogous computations one can prove that

$$
\begin{aligned}
\left\|\Phi_{2}(u)\right\|_{\infty} & \leq M+T^{2-\gamma_{2}}(M+1)^{p_{2}+q_{2}} \\
\left\|\Phi_{2}(u)-\Phi_{2}(\bar{u})\right\|_{\infty} & \leq C T^{2-\gamma_{2}}(M+1)^{p_{2}+q_{2}-1} d(u, \bar{u}) \\
\left\|\Phi_{2}(u)\right\|_{r_{2}} & \leq M+T^{2-\gamma_{2}-\frac{N}{2}\left(\frac{p_{2}}{\omega_{1}}+\frac{q_{2}}{\xi_{1}}-\frac{1}{r_{2}}\right)}(M+1)^{p_{2}+q_{2}}, \\
\left\|\Phi_{2}(u)-\Phi_{2}(\bar{u})\right\|_{r_{2}} & \leq C T^{2-\gamma_{2}-\frac{N}{2}\left(\frac{p_{2}}{\omega_{1}}+\frac{q_{2}}{\xi_{1}}-\frac{1}{r_{2}}\right)}(M+1)^{p_{2}+q_{2}-1} d(u, \bar{u}),
\end{aligned}
$$

if $T$ is suitably small such that $C T^{1-\gamma_{2}}(M+1)^{p_{2}+q_{2}-1} \leq 1 / 2$, we get the claimed result. Therefore the application $\Phi$ is a contraction in $K$ and by contraction mapping principle there exists a unique $(u, v) \in K$ satisfying $\Phi[(u, v)]=(u, v)$ and it is the solution to the semilinear problem (1.1).

\section{Proof of global existence theorem of the heat system}

\section{Proof of Theorem 3.3.}

This section is devoted to prove the first main result. To do this, let $\left(u_{0}, v_{0}\right) \in\left\{C_{0}\left(\mathbb{R}^{N}\right)\right\}^{2} \cap$ $L^{r_{1}}\left(\mathbb{R}^{N}\right) \times L^{r_{2}}\left(\mathbb{R}^{N}\right)$ where $r_{1}, r_{2}$ are given by (1.4). Let $(u, v)$ be a corresponding solution given by Theorem 3.2. Since $r_{1}>1$ and $r_{2}>1$ from (3.3), we have that

$$
(u, v) \in C\left(\left[0, T_{\max }\right), L^{r_{1}}\left(\mathbb{R}^{N}\right) \cap C_{0}\left(\mathbb{R}^{N}\right)\right) \times C\left(\left[0, T_{\max }\right), L^{r_{2}}\left(\mathbb{R}^{N}\right) \cap C_{0}\left(\mathbb{R}^{N}\right)\right)
$$

and (3.2) holds. Let us consider

$$
\begin{aligned}
& \varphi(t)=\|u(t)\|_{r_{1}}+(t+1)^{\frac{N}{2}\left(\frac{1}{r_{1}}-\frac{1}{\eta_{1}}\right)}\|u(t)\|_{\eta_{1}}+(t+1)^{\alpha}\|u(t)\|_{\infty}, \\
& \psi(t)=\|v(t)\|_{r_{2}}+(t+1)^{\frac{N}{2}\left(\frac{1}{r_{1}}-\frac{1}{w_{1}}\right)}\|v(t)\|_{w_{1}}+(t+1)^{\beta}\|v(t)\|_{\infty},
\end{aligned}
$$

be functions defined for $t \in\left[0, T_{\max }\right)$. We show that there exists $\varepsilon_{0}$ such that if $\varphi(0)+\psi(0) \leq \varepsilon_{0}$ and $T \in$ $\left(0, T_{\max }\right)$, then $\varphi, \psi$ are bounded on $[0, T]$, where $\alpha=\frac{\left(1-\gamma_{1}\right)\left(p_{2}-1\right)-\left(1-\gamma_{2}\right) q_{1}}{\left(\left(p_{1}-1\right)\left(p_{2}-1\right)-q_{1} q_{2}\right)}, \beta=\frac{\left(1-\gamma_{2}\right)\left(p_{1}-1\right)-\left(1-\gamma_{1}\right) q_{2}}{\left(\left(p_{1}-1\right)\left(p_{2}-1\right)-q_{1} q_{2}\right)}$ and $\eta_{1}, w_{1}>0$ are given by

$$
\frac{1}{\eta_{1}}=\frac{1}{r_{1}}-\frac{2}{N}[\alpha+\mu] ; \frac{1}{w_{1}}=\frac{1}{r_{2}}-\frac{2}{N}[\beta+\lambda]
$$

with $\mu, \lambda>0$ satisfying

$$
\begin{aligned}
& \mu<\min \left\{\gamma_{1}-\alpha, \frac{N}{2 r_{1}}-\alpha\right\}, \quad \lambda<\min \left\{\gamma_{2}-\beta, \frac{N}{2 r_{2}}-\beta\right\}, \\
& \frac{N}{2}\left(\frac{p_{1}}{r_{1}}+\frac{q_{1}}{r_{2}}-1\right)-\left(\alpha p_{1}+\beta q_{1}\right)<\left(p_{1} \mu+q_{1} \lambda\right)<1-\alpha, \\
& \frac{N}{2}\left(\frac{p_{2}}{r_{2}}+\frac{q_{2}}{r_{1}}-1\right)-\left(\beta p_{2}+\alpha q_{2}\right)<\left(p_{2} \lambda+q_{2} \mu\right)<1-\beta .
\end{aligned}
$$


If this statement is proved, then $T^{*}=\infty$ and $(u, v)$ is global. From (4.3)-(4.5), we have that

$$
\begin{aligned}
& \frac{p_{1}}{\eta_{1}}+\frac{q_{1}}{w_{1}}<1,0<\frac{N}{2}\left(\frac{p_{1}}{\eta_{1}}+\frac{q_{1}}{w_{1}}-\frac{1}{r_{1}}\right)<1, \\
& \frac{p_{2}}{w_{1}}+\frac{q_{2}}{\eta_{1}}<1,0<\frac{N}{2}\left(\frac{p_{2}}{w_{1}}+\frac{q_{2}}{\eta_{1}}-\frac{1}{r_{1}}\right)<1 .
\end{aligned}
$$

Moreover, from (3.3), it easy to check that

$$
\left\{\begin{array}{l}
\frac{p_{1}}{r_{1}}+\frac{q_{1}}{r_{2}}-\frac{2}{N}=\frac{2}{N}\left[\frac{\left(1-\gamma_{1}\right)\left(\left(p_{1}-1\right)\left(p_{2}-1\right)-q_{1} q_{2}\right)+\left(2-\gamma_{1}\right)\left(p_{2}-1\right)-\left(2-\gamma_{2}\right) q_{1}}{\left(\left(p_{1}-1\right)\left(p_{2}-1\right)-q_{1} q_{2}\right)}\right]<1 \\
\frac{p_{2}}{r_{2}}+\frac{q_{2}}{r_{1}}-\frac{2}{N}=\frac{2}{N}\left[\frac{\left(1-\gamma_{2}\right)\left(\left(p_{1}-1\right)\left(p_{2}-1\right)-q_{1} q_{2}\right)+\left(2-\gamma_{2}\right)\left(p_{1}-1\right)-\left(2-\gamma_{1}\right) q_{2}}{\left(\left(p_{1}-1\right)\left(p_{2}-1\right)-q_{1} q_{2}\right)}\right]<1 .
\end{array}\right.
$$

From the definition of $\alpha, \beta$, it yields

$$
\left\{\begin{array}{l}
q_{2} \alpha+\left(p_{2}-1\right) \beta=\left(1-\gamma_{2}\right) \\
q_{1} \beta+\left(p_{1}-1\right) \alpha=\left(1-\gamma_{1}\right)
\end{array}\right.
$$

An estimate for $(u, v)$ in $L^{r_{1}} \times L^{r_{2}}$. From (4.3) and (4.8), we have that

$$
\frac{p_{1}}{\eta_{1}}+\frac{q_{1}}{w_{1}}<\frac{p_{1}}{r_{1}}+\frac{q_{1}}{r_{2}}-\frac{2}{N}\left(\alpha p_{1}+q_{1} \beta\right)<\frac{2}{N}+\frac{1}{r_{1}}
$$

So, we can select $w \in\left(r_{2}, w_{1}\right)$ and $\xi \in\left(r_{1}, \eta_{1}\right)$ such that

$$
\max \left\{\frac{1}{r_{1}}, \frac{p_{1}}{\eta_{1}}+\frac{q_{1}}{w_{1}}, \frac{p_{1}}{r_{1}}+\frac{q_{1}}{r_{2}}-\frac{2}{N}\right\}<\frac{p_{1}}{\xi}+\frac{q_{1}}{w}<\min \left\{\frac{2}{N}+\frac{1}{r_{1}}, \frac{p_{1}}{r_{1}}+\frac{q_{1}}{r_{2}}, 1\right\} .
$$

To see this, let us take $\frac{1}{\xi}=\frac{(1-k)}{\eta_{1}}+\frac{k}{r_{1}}, \frac{1}{w}=\frac{(1-k)}{w_{1}}+\frac{k}{r_{2}}, 0<k<1$,

$$
\frac{p_{1}}{\xi}+\frac{q_{1}}{w}=\frac{p_{1}}{\eta_{1}}+\frac{q_{1}}{w_{1}}+k\left(\frac{p_{1}}{r_{1}}+\frac{q_{1}}{r_{2}}-\left(\frac{p_{1}}{\eta_{1}}+\frac{q_{1}}{w_{1}}\right)\right) .
$$

It's easy to check that for some $0<k<1$ the inequality (4.9) holds.

Observe that by (4.9)

$$
0<\frac{N}{2}\left(\frac{p_{1}}{\xi}+\frac{q_{1}}{w}-\frac{1}{r_{1}}\right)<1,0<\frac{N}{2}\left(\frac{p_{1}}{r_{1}}+\frac{q_{1}}{r_{2}}-\left(\frac{p_{1}}{\xi}+\frac{q_{1}}{w}\right)\right)<1 .
$$

Making use of the interpolation inequality, we get

$$
\begin{gathered}
\|u\|_{\xi} \leq\|u\|_{r_{1}}^{\theta}\|u\|_{\eta_{1}}^{1-\theta} \leq\|u\|_{r_{1}}^{\theta}\left[(t+1)^{\frac{N}{2}\left(\frac{1}{r_{1}}-\frac{1}{\eta_{1}}\right)}\|u\|_{\eta_{1}}\right]^{1-\theta}(t+1)^{\frac{N}{2}\left(\frac{1}{r_{1}}-\frac{1}{\xi}\right)} \\
\|v\|_{w} \leq\|v\|_{r_{2}}^{\theta}\|v\|_{w_{1}}^{1-\theta} \leq\|v\|_{r_{2}}^{\theta}\left[(t+1)^{\frac{N}{2}\left(\frac{1}{r_{2}}-\frac{1}{w_{1}}\right)}\|v\|_{w_{1}}\right]^{1-\theta}(t+1)^{\frac{N}{2}\left(\frac{1}{r_{2}}-\frac{1}{w}\right)}
\end{gathered}
$$

where $\frac{1}{\xi}=\frac{\theta}{r_{1}}+\frac{1-\theta}{\eta_{1}}, \frac{1}{w}=\frac{\theta^{\prime}}{r_{2}}+\frac{1-\theta^{\prime}}{w_{1}}, \theta, \theta^{\prime} \in(0,1)$. We easily check that

$$
2-\gamma_{1}-\frac{N}{2}\left(\frac{p_{1}}{\xi}+\frac{q_{1}}{w}-\frac{1}{r_{1}}\right)-\frac{N}{2}\left(\frac{p_{1}}{r_{1}}+\frac{q_{1}}{r_{2}}-\left(\frac{p_{1}}{\xi}+\frac{q_{1}}{w}\right)\right)=0,
$$

we see from (3.1), (4.10) and (4.11) that

$$
\|u(t)\|_{r_{1}} \leq\left\|u_{0}\right\|_{r_{1}}+\int_{0}^{t}(t-s)^{-\frac{N}{2}\left(\frac{p_{1}}{\xi}+\frac{q_{1}}{w}-\frac{1}{r_{1}}\right)} \int_{0}^{s}(s-\tau)^{-\gamma_{1}}\|u(\tau)\|_{\xi}^{p_{1}}\|v(\tau)\|_{w}^{q_{1}} d \tau d s
$$




$$
\begin{aligned}
& \leq\left\|u_{0}\right\|_{r_{1}}+\int_{0}^{t}(t-s)^{-\frac{N}{2}\left(\frac{p_{1}}{\xi}+\frac{q_{1}}{w}-\frac{1}{r_{1}}\right)} \int_{0}^{s}(s-\tau)^{-\gamma_{1}} \tau^{-\frac{N}{2}\left(\frac{p_{1}}{r_{1}}+\frac{q_{1}}{r_{2}}-\left(\frac{p_{1}}{\xi}+\frac{q_{1}}{w}\right)\right)} d \tau d s \\
& \leq\left\|u_{0}\right\|_{r_{1}}+C\left[\sup _{s \in(0, t)} \varphi(s)\right]^{p_{1}}\left[\sup _{s \in(0, t)} \psi(s)\right]^{q_{1}} .
\end{aligned}
$$

An estimate for $(u, v)$ in $L^{\eta_{1}} \times L^{w_{1}}$. From (3.5) as $\frac{p_{1}}{r_{1}}+\frac{q_{1}}{r_{2}}-\frac{2}{N}<\frac{2}{N}+\frac{1}{\eta_{1}}, \frac{p_{1}}{r_{1}}+\frac{q_{1}}{r_{2}}<1+\frac{2}{N}$. Since

$$
p_{1} \mu+q_{1} \lambda>\mu=2-\gamma_{1}-\left(1-\gamma_{1}\right)-1+\mu=\frac{N}{2}\left(\frac{p_{1}-1}{r_{1}}+\frac{q_{1}}{r_{2}}\right)-\left(p_{1}-1\right) \alpha-q_{1} \beta-1+\mu
$$

which equivalently

$$
\frac{N}{2}\left(\frac{p_{1}-1}{r_{1}}+\frac{q_{1}}{r_{2}}\right)-\left(p_{1}-1\right)[\alpha+\mu]-q_{1}[\beta+\lambda]<1
$$

that is

$$
\frac{p_{1}-1}{r_{1}}-\frac{2\left(p_{1}-1\right)}{N}[\alpha+\mu]+\frac{q_{1}}{r_{2}}-\frac{2 q_{1}}{N}[\beta+\lambda]<\frac{2}{N}
$$

Namely $\frac{p_{1}-1}{\eta_{1}}+\frac{q_{1}}{w_{1}}<\frac{2}{N}$. From these facts, we can choose $w^{\prime} \in\left(r_{2}, w_{1}\right), \xi^{\prime} \in\left(r_{1}, \eta_{1}\right)$ satisfying

$$
\max \left\{\frac{1}{\eta_{1}}, \frac{p_{1}}{\eta_{1}}+\frac{q_{1}}{w_{1}}, \frac{p_{1}}{r_{1}}+\frac{q_{1}}{r_{2}}-\frac{2}{N}\right\}<\frac{p_{1}}{\xi^{\prime}}+\frac{q_{1}}{w^{\prime}}<\min \left\{\frac{2}{N}+\frac{1}{\eta_{1}}, \frac{p_{1}}{r_{1}}+\frac{q_{1}}{r_{2}}, 1\right\} .
$$

Note that

$$
0<\frac{N}{2}\left(\frac{p_{1}}{r_{1}}+\frac{q_{1}}{r_{2}}-\left(\frac{p_{1}}{\xi^{\prime}}+\frac{q_{1}}{w^{\prime}}\right)\right)<1,0<\frac{N}{2}\left(\frac{p_{1}}{\xi^{\prime}}+\frac{q_{1}}{w^{\prime}}-\frac{1}{\eta_{1}}\right)<1
$$

and

$$
2-\gamma_{1}+\frac{N}{2}\left(\frac{1}{r_{1}}-\frac{1}{\eta_{1}}\right)-\frac{N}{2}\left(\frac{p_{1}}{\xi^{\prime}}+\frac{q_{1}}{w^{\prime}}-\frac{1}{\eta_{1}}\right)-\frac{N}{2}\left(\left(\frac{p_{1}}{r_{1}}+\frac{q_{1}}{r_{2}}\right)-\left(\frac{p_{1}}{\xi^{\prime}}+\frac{q_{1}}{w^{\prime}}\right)\right)=0 .
$$

From (2.1), (2.2), (4.11) and (4.12), we get

$$
\begin{aligned}
\|u(t)\|_{\eta_{1}} \leq & (t+1)^{-\frac{N}{2}\left(\frac{1}{r_{1}}-\frac{1}{\eta_{1}}\right)}\left(\left\|u_{0}\right\|_{r_{1}}+\left\|u_{0}\right\|_{\eta_{1}}\right) \\
& +\int_{0}^{t}(t-s)^{-\frac{N}{2}\left(\frac{p_{1}}{\xi^{\prime}}+\frac{q_{1}}{w^{\prime}}-\frac{1}{\eta_{1}}\right)} \int_{0}^{s}(s-\tau)^{-\gamma_{1}}\|u(\tau)\|_{\xi^{\prime}}^{p_{1}}\|v(\tau)\|_{w^{\prime}}^{q_{1}} d \tau d s \\
\leq & (t+1)^{-\frac{N}{2}\left(\frac{1}{r_{1}}-\frac{1}{\eta_{1}}\right)}\left(\left\|u_{0}\right\|_{r_{1}}+\left\|u_{0}\right\|_{\eta_{1}}\right)+C\left[\sup _{s \in(0, t)} \varphi(s)\right]^{p_{1}}\left[\sup _{s \in(0, t)} \psi(s)\right]^{q_{1}} \\
& \times \int_{0}^{t}(t-s)^{-\frac{N}{2}\left(\frac{p_{1}}{\xi^{t}}+\frac{q_{1}}{w^{\prime}}-\frac{1}{\eta_{1}}\right)} \int_{0}^{s}(s-\tau)^{-\gamma_{1}} \tau^{-\frac{N}{2}\left(\frac{p_{1}}{r_{1}}+\frac{q_{1}}{r_{2}}-\left(\frac{p_{1}}{\xi^{\prime}}+\frac{q_{1}}{w^{\prime}}\right)\right)} d \tau d s,
\end{aligned}
$$

which, yields

$$
(t+1)^{-\frac{N}{2}\left(\frac{1}{r_{1}}-\frac{1}{\eta_{1}}\right)}\left\|u_{1}(t)\right\|_{\eta_{1}} \leq\left\|u_{0}\right\|_{r_{1}}+\left\|u_{0}\right\|_{\eta_{1}}+C\left[\sup _{s \in(0, t)} \varphi(s)\right]^{p_{1}}\left[\sup _{s \in(0, t)} \psi(s)\right]^{q_{1}}
$$


Estimate for $\|u(t)\|_{\infty}$. We have to distinguish two situations

Case a: Either $N \leq 2$ or $\left(\frac{p_{1}}{r_{1}}+\frac{q_{1}}{r_{2}}<\frac{4}{N}\right.$ and $\left.\frac{p_{1}}{\eta_{1}}+\frac{q_{1}}{w_{1}}<\frac{2}{N}\right)$. From (4.3),(4.6) and (4.7), there exist $w^{\prime \prime} \in\left(r_{2}, w_{1}\right), \eta^{\prime \prime} \in\left(r_{1}, \eta_{1}\right)$ such that

$$
\max \left\{\frac{p_{1}}{r_{1}}+\frac{q_{1}}{r_{2}}-\frac{2}{N}, \frac{p_{1}}{\eta_{1}}+\frac{q_{1}}{w_{1}}\right\}<\left(\frac{p_{1}}{\eta^{\prime \prime}}+\frac{q_{1}}{w^{\prime \prime}}\right)<\min \left\{\frac{2}{N}, \frac{p_{1}}{r_{1}}+\frac{q_{1}}{r_{2}}, 1\right\} .
$$

Since $w^{\prime \prime} \in\left(r_{2}, w_{1}\right), \eta^{\prime \prime} \in\left(r_{1}, \eta_{1}\right)$, using interpolation inequality again as in (4.11), we obtain

$$
\begin{aligned}
\|u(t)\|_{\infty} \leq & (t+1)^{-\frac{N}{2} \frac{1}{r_{1}}}\left(\left\|u_{0}\right\|_{r_{1}}+\left\|u_{0}\right\|_{\infty}\right)+\int_{0}^{t}(t-s)^{-\frac{N}{2}\left(\frac{q_{1}}{w^{\prime \prime}}+\frac{p_{1}}{\eta^{\prime}}\right)} \int_{0}^{s}(s-\tau)^{-\gamma_{1}} \\
& \times\|u(\tau)\|_{\eta^{\prime \prime}}^{p_{1}}\|v(\tau)\|_{w^{\prime \prime}}^{q_{1}} d \tau d s \\
\leq & (t+1)^{-\frac{N}{2} \frac{1}{r_{1}}}\left(\left\|u_{0}\right\|_{r_{1}}+\left\|u_{0}\right\|_{\infty}\right)+C\left[\sup _{s \in(0, t)} \varphi(s)\right]^{p_{1}}\left[\sup _{s \in(0, t)} \psi(s)\right]^{q_{1}} \\
& \times \int_{0}^{t}(t-s)^{-\frac{N}{2}\left(\frac{q_{1}}{w^{\prime \prime}}+\frac{p_{7}}{\eta^{\prime}}\right)} \int_{0}^{s}(s-\tau)^{-\gamma_{1}}(\tau+1)^{-\frac{N}{2}\left(\frac{p_{1}}{r_{1}}+\frac{q_{1}}{r_{2}}-\left(\frac{p_{1}}{\eta^{\prime}}+\frac{q_{1}}{w^{\prime \prime}}\right)\right)} d \tau d s .
\end{aligned}
$$

Notice that $0<\frac{N}{2}\left(\frac{p_{1}}{r_{1}}+\frac{q_{1}}{r_{2}}-\left(\frac{p_{1}}{\eta^{\prime \prime}}+\frac{q_{1}}{w^{\prime \prime}}\right)\right)<1, \frac{N}{2}\left(\frac{q_{1}}{w^{\prime \prime}}+\frac{p_{1}}{\eta^{\prime \prime}}\right)<1$. On the other hand, since $\alpha-\frac{N}{2 r_{1}}=$ $\frac{p_{2}-q_{1}-1}{\left(\left(p_{1}-1\right)\left(p_{2}-1\right)-q_{1} q_{2}\right)}<0$, it follows that

$$
\begin{aligned}
& \alpha+2-\gamma_{1}-\frac{N}{2}\left(\frac{q_{1}}{w^{\prime \prime}}+\frac{p_{1}}{\eta^{\prime \prime}}\right)-\frac{N}{2}\left(\left(\frac{p_{1}}{r_{1}}+\frac{q_{1}}{r_{2}}\right)-\left(\frac{p_{1}}{\eta^{\prime \prime}}+\frac{q_{1}}{w^{\prime \prime}}\right)\right) \\
< & 2-\gamma_{1}+\frac{N}{2 r_{1}}-\frac{N}{2}\left[\frac{p_{1}}{r_{1}}+\frac{q_{1}}{r_{2}}\right]=0,
\end{aligned}
$$

which, together with (2.5), yields

$$
(t+1)^{\alpha}\|u(t)\|_{\infty} \leq\left(\left\|u_{0}\right\|_{r_{1}}+\left\|u_{0}\right\|_{\infty}\right)+C\left[\sup _{s \in(0, t)} \varphi(s)\right]^{p_{1}}\left[\sup _{s \in(0, t)} \psi(s)\right]^{q_{1}} .
$$

Or else, that is $N>2$ and $\left(\frac{p_{1}}{r_{1}}+\frac{q_{1}}{r_{2}} \geq \frac{4}{N}\right.$, or $\left.\frac{p_{1}}{\eta_{1}}+\frac{q_{1}}{w_{1}} \geq \frac{2}{N}\right)$. From (4.3),(4.6) and (4.7), there exist $w^{\prime \prime} \in\left(r_{2}, w_{1}\right), \xi^{\prime \prime} \in\left(r_{1}, \eta_{1}\right)$ such that

$$
\max \left\{\frac{p_{1}}{r_{1}}+\frac{q_{1}}{r_{2}}-\frac{2}{N}, \frac{p_{1}}{\eta_{1}}+\frac{q_{1}}{w_{1}}, \frac{2}{N}\right\}<\left(\frac{p_{1}}{\xi^{\prime \prime}}+\frac{q_{1}}{w^{\prime \prime}}\right)<\min \left\{1, \frac{p_{1}}{r_{1}}+\frac{q_{1}}{r_{2}}\right\} .
$$

Hence, the inequalities (2.2) and (4.11), give

$$
\begin{aligned}
& \|u(t)\|_{\infty} \leq(t+1)^{-\frac{N}{2} \frac{1}{r_{1}}}\left(\left\|u_{0}\right\|_{r_{1}}+\left\|u_{0}\right\|_{\infty}\right) \\
& +\int_{0}^{t}(t-s+1)^{-\frac{N}{2}\left(\frac{p_{1}}{\xi^{\prime}}+\frac{q_{1}}{w^{\prime \prime}}\right)} \int_{0}^{s}(s-\tau)^{-\gamma_{1}}\left(\|u(\tau)\|_{\xi^{\prime \prime}}^{p_{1}}\|v(\tau)\|_{w^{\prime \prime}}^{q_{1}}+\|u(\tau)\|_{\infty}^{p_{1}}\|v(\tau)\|_{\infty}^{q_{1}}\right) d \tau d s .
\end{aligned}
$$


Therefore

$$
\begin{aligned}
\|u(t)\|_{\infty} \leq & (t+1)^{-\frac{N}{2} \frac{1}{r_{1}}}\left(\left\|u_{0}\right\|_{r_{1}}+\left\|u_{0}\right\|_{\infty}\right) \\
& +\left[\sup _{s \in(0, t)} \varphi(s)\right]^{p_{1}}\left[\sup _{s \in(0, t)} \psi(s)\right]^{q_{1}} \int_{0}^{t}(t-s+1)^{-\frac{N}{2}\left(\frac{p_{1}}{\xi^{\prime \prime}}+\frac{q_{1}}{w^{\prime \prime}}\right)} \int_{0}^{s}(s-\tau)^{-\gamma_{1}} \\
& +\left[\sup _{s \in(0, t)} \varphi(s)\right]^{p_{1}}\left[\sup _{s \in(0, t)} \psi(s)\right]^{q_{1}} \int_{0}^{t}(t-s+1)^{-\frac{N}{2}\left(\frac{p_{1}}{\xi^{\prime \prime}}+\frac{q_{1}}{w^{\prime \prime}}\right)} \int_{0}^{s}(s-\tau)^{-\gamma_{1}} \times \\
& {\left[(\tau+1)^{-\frac{N}{2}\left(\frac{p_{1}}{r_{1}}+\frac{q_{1}}{r_{2}}-\left(\frac{p_{1}}{\xi^{\prime \prime}}+\frac{q_{1}}{w^{\prime}}\right)\right)}+(\tau+1)^{-\left(\alpha p_{1}+\beta q_{1}\right)}\right] d \tau d s . }
\end{aligned}
$$

Since $\alpha p_{1}+\beta q_{1}<1$, we observe that

$$
\frac{p_{1}}{r_{1}}+\frac{q_{1}}{r_{2}}-\frac{2}{N}<\frac{p_{1}}{r_{1}}+\frac{q_{1}}{r_{2}}-\frac{2}{N}\left(\alpha p_{1}+q_{1} \beta\right) .
$$

It easy to see from (4.3), (4.7) and (4.8) that

$$
\max \left\{\frac{p_{1}}{\eta_{1}}+\frac{q_{1}}{w_{1}}, \frac{2}{N}\right\}<\frac{p_{1}}{r_{1}}+\frac{q_{1}}{r_{2}}-\frac{2}{N}\left(\alpha p_{1}+q_{1} \beta\right) .
$$

From these facts, we can assume that $\left(\frac{p_{1}}{\xi^{\prime \prime}}+\frac{q_{1}}{w^{\prime \prime}}\right) \leq \frac{p_{1}}{r_{1}}+\frac{q_{1}}{r_{2}}-\frac{2}{N}\left(\alpha p_{1}+q_{1} \beta\right)$. Thus

$$
\alpha+1-\gamma_{1}-\frac{N}{2}\left(\frac{p_{1}}{r_{1}}+\frac{q_{1}}{r_{2}}\right)+\frac{N}{2}\left(\frac{p_{1}}{\xi^{\prime \prime}}+\frac{q_{1}}{w^{\prime \prime}}\right)=\alpha p_{1}+q_{1} \beta-\frac{N}{2}\left(\frac{p_{1}}{r_{1}}+\frac{q_{1}}{r_{2}}-\left(\frac{p_{1}}{\xi^{\prime \prime}}+\frac{q_{1}}{w^{\prime \prime}}\right)\right) \leq 0,
$$

and therefore, we conclude as in the previous case

$$
(t+1)^{\alpha}\|u(t)\|_{\infty} \leq C\left(\left\|u_{0}\right\|_{r_{1}}+\left\|u_{0}\right\|_{\infty}\right)+C\left[\sup _{s \in(0, t)} \varphi(s)\right]^{p_{1}}\left[\sup _{s \in(0, t)} \psi(s)\right]^{q_{1}} .
$$

From the previous estimates of $\left\|u_{1}(t)\right\|_{r_{1}},\left\|u_{1}(t)\right\|_{\eta_{1}}$ and $\|u(t)\|_{\infty}$, we obtain

$$
\varphi(t) \leq C\left(\left\|u_{0}\right\|_{r_{1}}+\left\|u_{0}\right\|_{\infty}\right)+C\left[\sup _{s \in(0, t)} \varphi(s)\right]^{p_{1}}\left[\sup _{s \in(0, t)} \psi(s)\right]^{q_{1}} .
$$

By analogous computations, we get $\psi(t) \leq C\left(\left\|v_{0}\right\|_{r_{1}}+\left\|v_{0}\right\|_{\infty}\right)+C\left[\sup _{s \in(0, t)} \psi(s)\right]^{p_{2}}\left[\sup _{s \in(0, t)} \varphi(s)\right]^{q_{2}}$. Denoting $f(t)=\sup _{s \in(0, t)} \varphi(s)$ and $g(t)=\sup _{s \in(0, t)} \psi(s)$, we get

$$
\begin{aligned}
& f(t) \leq C\left(\left\|u_{0}\right\|_{r_{1}}+\left\|u_{0}\right\|_{\infty}\right)+C f(t)^{p_{1}} g(t)^{q_{1}}, \\
& g(t) \leq C\left(\left\|v_{0}\right\|_{r_{2}}+\left\|v_{0}\right\|_{\infty}\right)+C g(t)^{p_{2}} f(t)^{q_{2}} .
\end{aligned}
$$

We now define $h(t):=f(t)+g(t)$. Taking into account (4.15), (4.16) reads

$$
h(t) \leq C\left(\varepsilon+h^{p_{1}+q_{1}}(t)+h^{p_{2}+q_{2}}(t)\right), \forall t \in\left[0, T_{\max }\right),
$$

for some positive constant $C$ independent of $t$ and $A=\left\|u_{0}\right\|_{r_{1}}+\left\|u_{0}\right\|_{\infty}+\left\|v_{0}\right\|_{r_{2}}+\left\|v_{0}\right\|_{\infty}<\varepsilon$. We conclude by standard arguments for sufficiently small $\varepsilon$ as in [32], it then follows that $h(t) \leq C \varepsilon, \forall$ $t \in\left[0, T_{\max }\right)$. Hence $f(t) \leq C \varepsilon, g(t) \leq C \varepsilon, \forall t \in\left[0, T_{\max }\right)$. 


\section{Damped wave system}

Similar considerations to the system for heat equations can be applied to the Cauchy problem (3.6) for the system of damped wave equations in low dimensional space.

\subsection{Proof of theorem 3.7}

We follow the same steps as in the proof of Theorem 3.3 with a slight modifications. So we maintain some notations used in the previous proof.

Let us define $\eta_{1}, w_{1}>0$ by

$$
\frac{1}{\eta_{1}}=\frac{1}{r_{1}}-\frac{2}{N}(\alpha+\mu) ; \quad \frac{1}{w_{1}}=\frac{1}{r_{2}}-\frac{2}{N}(\beta+\lambda)
$$

with $\mu, \lambda>0$ satisfying

$$
\begin{aligned}
& \mu<\min \left\{\gamma_{1}-\alpha, \frac{N}{2 r_{1}}-\alpha\right\}, \quad \lambda<\min \left\{\gamma_{2}-\beta, \frac{N}{2 r_{2}}-\beta\right\}, \\
& \frac{N}{2}\left(\frac{p_{1}}{r_{1}}+\frac{q_{1}}{r_{2}}-1\right)-\left(\alpha p_{1}+\beta q_{1}\right)<\left(p_{1} \mu+q_{1} \lambda\right)<1-\alpha, \\
& \frac{N}{2}\left(\frac{p_{2}}{r_{2}}+\frac{q_{2}}{r_{1}}-1\right)-\left(\beta p_{2}+\alpha q_{2}\right)<\left(p_{2} \lambda+q_{2} \mu\right)<1-\beta,
\end{aligned}
$$

with

$$
\mu=\frac{\left(p_{2}-q_{1}-1\right)}{p_{1}-q_{2}-1} \lambda
$$

The existence of $\lambda$ and $\mu$ are insured by the conditions (3.11)-(3.13).

Let $\left(u_{i}, v_{i}\right) \in\left\{W^{1-i, 1}\left(\mathbb{R}^{N}\right) \times W^{1-i, \infty}\left(\mathbb{R}^{N}\right)\right\}^{2}, i=0,1$. From (4.2), we have $r_{1}>1$ and $r_{2}>1$.

Let $(u, v)$ be a corresponding solution of (3.6) given by Proposition 3.5.

Ou aim is to seek upper bound of solution in the functionals defined in (4.1), (4.2) each $t>0$. It easy to check that all the requirements (4.6), (4.7) and (4.8) are fulfilled.

An estimate for $u$ in $L^{r_{1}}\left(\mathbb{R}^{N}\right)$. From (4.8), we conclude that

$$
\frac{p_{1}}{\eta_{1}}+\frac{q_{1}}{w_{1}}<\frac{p_{1}}{r_{1}}+\frac{q_{1}}{r_{2}}-\frac{2}{N}\left(\alpha p_{1}+q_{1} \beta\right)<\frac{2}{N}+\frac{1}{r_{1}}
$$

So, we can select $w \in\left(r_{2}, w_{1}\right)$ and $\eta \in\left(r_{1}, \eta_{1}\right)$ such that

$$
\max \left\{\frac{1}{r_{1}}, \frac{p_{1}}{\eta_{1}}+\frac{q_{1}}{w_{1}}, \frac{p_{1}}{r_{1}}+\frac{q_{1}}{r_{2}}-\frac{2}{N}\right\}<\frac{p_{1}}{\eta}+\frac{q_{1}}{w}<\min \left\{\frac{2}{N}+\frac{1}{r_{1}}, \frac{p_{1}}{r_{1}}+\frac{q_{1}}{r_{2}}, 1\right\} .
$$

Note that

$$
0 \leq \frac{N}{2}\left(\frac{p_{1}}{\eta}+\frac{q_{1}}{w}-\frac{1}{r_{1}}\right)<1,0 \leq \frac{N}{2}\left(\frac{p_{1}}{r_{1}}+\frac{q_{1}}{r_{2}}-\left(\frac{p_{1}}{\eta}+\frac{q_{1}}{w}\right)\right)<1 .
$$


and by (3.11)-(3.13), we get

$$
\frac{1}{r_{1}}<\frac{p_{1}}{\eta_{1}}+\frac{q_{1}}{w_{1}}, \frac{1}{\eta_{1}}<\frac{p_{1}}{\eta_{1}}+\frac{q_{1}}{w_{1}}, \frac{1}{w_{1}}<\frac{p_{2}}{\eta_{1}}+\frac{q_{2}}{w_{1}}, \text { and } \frac{1}{\eta_{1}}<\frac{p_{2}}{\eta_{1}}+\frac{q_{2}}{w_{1}} .
$$

From (3.10), we have

$$
\begin{aligned}
\|u(t, .)\|_{r_{1}} & \leq\left\|\left(K_{1}(t)-e^{-\frac{t}{2}} W_{1}(t)\right)\left(\frac{1}{2} u_{0}+u_{1}\right)\right\|_{r_{1}}+\left\|e^{-\frac{t}{2}} W_{1}(t)\left(\frac{1}{2} u_{0}+u_{1}\right)\right\|_{r_{1}} \\
& +\left\|\left(K_{0}(t)-e^{-\frac{t}{2}}\left(W_{0}(t)+\frac{t}{8} W_{1}(t)\right)\right) u_{0}\right\|_{r_{1}}+\left\|e^{-\frac{t}{2}}\left(W_{0}(t)+\frac{t}{8} W_{1}(t)\right) u_{0}\right\|_{r_{1}} \\
& +\int_{0}^{t}\left\|\left(K_{1}(t-s)-e^{-\frac{t-s}{2}} W_{1}(t-s)\right) \int_{0}^{s}(s-\tau)^{-\gamma_{1}}|u(\tau)|^{p_{1}}|v(\tau)|^{q_{1}}\right\|_{r_{1}} d \tau d s \\
& +\int_{0}^{t} e_{r_{1}}^{-\frac{t-s}{2}}\left\|W_{1}(t-s) \int_{0}^{s}(s-\tau)^{-\gamma_{1}}|u(\tau)|^{p_{1}}|v(\tau)|^{q_{1}}\right\|_{r_{1}} d \tau d s .
\end{aligned}
$$

Next, by the lemma 2.4, lemma 2.5, and (2.3) we get, for all $t \in\left[0, T_{\max }\right)$

$$
\begin{aligned}
\|u(t, .)\|_{r_{1}} & \leq C\left(\left\|u_{1}\right\|_{L^{r_{1}}}+\left\|u_{0}\right\|_{W^{1, r_{1}}}\right)+\int_{0}^{t}(1+t-s)^{-\frac{N}{2}\left(\frac{p_{1}}{\eta}+\frac{q_{1}}{w}-\frac{1}{r_{1}}\right)} \\
& \times \int_{0}^{s}(s-\tau)^{-\gamma_{1}}\|u(\tau, .)\|_{\eta}^{p_{1}}\|v(\tau, .)\|_{w}^{q_{1}} d \tau d s \\
& +\int_{0}^{t} e^{-\frac{t-s}{2}}(t-s) \int_{0}^{s}(s-\tau)^{-\gamma_{1}}\left\||u(\tau)|^{p_{1}}|v(\tau)|^{q_{1}}\right\|_{r_{1}} d \tau d s .
\end{aligned}
$$

By using the interpolation inequality to the last term, we get

$$
\begin{aligned}
& \|u(t, .)\|_{r_{1}} \leq C\left(\left\|u_{1}\right\|_{r_{1}}+\left\|u_{0}\right\|_{W^{1, r_{1}}}\right) \\
& \quad+\int_{0}^{t}(t-s+1)^{-\frac{N}{2}\left(\frac{p_{1}}{\eta}+\frac{q_{1}}{w}-\frac{1}{r_{1}}\right)} \int_{0}^{s}(s-\tau)^{-\gamma_{1}}\|u(\tau, .)\|_{\eta}^{p_{1}}\|v(\tau, .)\|_{w}^{q_{1}} d \tau d s \\
& \quad+\int_{0}^{t} e^{-\frac{t-s}{2}}(t-s) \int_{0}^{s}(s-\tau)^{-\gamma_{1}}\left\|\left.u(\tau)\right|^{p_{1}}|v(\tau)|^{q_{1}}\right\|_{\left(\frac{p_{1}}{\eta_{1}}+\frac{q_{1}}{w_{1}}\right)^{-1}\left\|\left.u(\tau)\right|^{p_{1}}|v(\tau)|^{q_{1}}\right\|_{\infty}^{\theta} d \tau d s}
\end{aligned}
$$

with $1-\theta=\frac{1}{r_{1}}\left(\frac{p_{1}}{\eta_{1}}+\frac{q_{1}}{w_{1}}\right)^{-1}$. Therefore

$$
\begin{aligned}
& \|u(t, .)\|_{r_{1}} \leq C\left(\left\|u_{1}\right\|_{r_{1}}+\left\|u_{0}\right\|_{W^{1, r_{1}}}\right) \\
& \quad+\int_{0}^{t}(t-s+1)^{-\frac{N}{2}\left(\frac{p_{1}}{\eta}+\frac{q_{1}}{w}-\frac{1}{r_{1}}\right)} \int_{0}^{s}(s-\tau)^{-\gamma_{1}}\|u(\tau, .)\|_{\eta}^{p_{1}}\|v(\tau, .)\|_{w}^{q_{1}} d \tau d s
\end{aligned}
$$




$$
+\int_{0}^{t} e^{-\frac{t-s}{2}}(t-s) \int_{0}^{s}(s-\tau)^{-\gamma_{1}}\|u(\tau, .)\|_{\eta_{1}}^{p_{1}(1-\theta)}\|v(\tau, .)\|_{w_{1}}^{q_{1}(1-\theta)}\|u(\tau, .)\|_{\infty}^{p_{1} \theta}\|v(\tau, .)\|_{\infty}^{q_{1} \theta} d \tau d s
$$

From the definition of $\varphi$ and $\psi$, we infer that

$$
\begin{aligned}
\|u(t, .)\|_{r_{1}} \leq & C\left(\left\|u_{1}\right\|_{r_{1}}+\left\|u_{0}\right\|_{W^{1, r_{1}}}\right)+\left[\sup _{s \in(0, t)} \varphi(s)\right]^{p_{1}}\left[\sup _{s \in(0, t)} \psi(s)\right]^{q_{1}} \\
& \times \int_{0}^{t}(t-s+1)^{-\frac{N}{2}\left(\frac{p_{1}}{\eta}+\frac{q_{1}}{w}-\frac{1}{r_{1}}\right)} \int_{0}^{s}(s-\tau)^{-\gamma_{1}} \tau^{-\frac{N}{2}\left(\frac{p_{1}}{r_{1}}+\frac{q_{1}}{r_{2}}-\left(\frac{p_{1}}{\eta}+\frac{q_{1}}{w}\right)\right)} d \tau d s \\
& +\int_{0}^{t} e^{-\frac{t-s}{2}}(t-s) \int_{0}^{s}(s-\tau)^{-\gamma_{1}}(1+\tau)^{-\left(\alpha p_{1}+\beta q_{1}\right) \theta-\frac{N}{2}\left(\frac{p_{1}}{r_{1}}+\frac{q_{1}}{r_{2}}-\left(\frac{p_{1}}{\eta_{1}}+\frac{q_{1}}{w_{1}}\right)\right)(1-\theta)} d \tau d s \\
& \times\left[\sup _{s \in(0, t)} \varphi(s)\right]^{p_{1}}\left[\sup _{s \in(0, t)} \psi(s)\right]^{q_{1}}
\end{aligned}
$$

where we have have used the fact that (4.1) and (4.11) since $(u, v) \in X$.

By virtue of (4.8), we know that

$$
\begin{aligned}
& 1-\gamma_{1}-\left(\alpha p_{1}-\beta q_{1}\right) \theta-\frac{N}{2}\left(\frac{p_{1}}{r_{1}}+\frac{q_{1}}{r_{2}}-\left(\frac{p_{1}}{\eta_{1}}+\frac{q_{1}}{w_{1}}\right)\right)(1-\theta) \\
= & 1-\gamma_{1}-\left(\alpha p_{1}-\beta q_{1}\right) \theta-\left(p_{1}(\alpha+\mu)+q_{1}(\beta+\lambda)\right)(1-\theta) \\
= & -\alpha-\left(p_{1} \mu+q_{1} \lambda\right)(1-\theta)<0
\end{aligned}
$$

and since $2-\gamma_{1}-\frac{N}{2}\left(\frac{p_{1}}{\eta}+\frac{q_{1}}{w}-\frac{1}{r_{1}}\right)-\frac{N}{2}\left(\frac{p_{1}}{r_{1}}+\frac{q_{1}}{r_{2}}-\left(\frac{p_{1}}{\eta}+\frac{q_{1}}{w}\right)\right)=0$. Applying again Lemma 2.2, we obtain

$$
\|u(t, .)\|_{r_{1}} \leq C\left(\left\|u_{0}\right\|_{W^{1, r_{1}}}+\left\|u_{1}\right\|_{r_{1}}\right)+C\left[\sup _{s \in(0, t)} \varphi(s)\right]^{p_{1}}\left[\sup _{s \in(0, t)} \psi(s)\right]^{q_{1}} .
$$

An estimate for $\|u(t, .)\|_{\eta_{1}}$. Arguing in the same way as in the previous estimate

$$
\begin{aligned}
\|u(t, .)\|_{\eta_{1}} & \leq\left\|\left(K_{1}(t)-e^{-\frac{t}{2}} W_{1}(t)\right)\left(\frac{1}{2} u_{0}+u_{1}\right)\right\|_{\eta_{1}}+\left\|e^{-\frac{t}{2}} W_{1}(t)\left(\frac{1}{2} u_{0}+u_{1}\right)\right\|_{\eta_{1}} \\
& +\left\|\left(K_{0}(t)-e^{-\frac{t}{2}}\left(W_{0}(t)+\frac{t}{8} W_{1}(t)\right)\right) u_{0}\right\|_{\eta_{1}}+\left\|e^{-\frac{t}{2}}\left(W_{0}(t)+\frac{t}{8} W_{1}(t)\right) u_{0}\right\|_{\eta_{1}} \\
& +\int_{0}^{t}\left\|\left(K_{1}(t-s)-e^{-\frac{t-s}{2}} W_{1}(t-s)\right) \int_{0}(s-\tau)^{-\gamma_{1}}|u(\tau)|^{p_{1}}|v(\tau)|^{q_{1}}\right\|_{\eta_{1}} d \tau d s \\
& +\int_{0}^{t} e^{-\frac{t-s}{2}}\left\|W_{1}(t-s) \int_{0}^{s}(s-\tau)^{-\gamma_{1}}|u(\tau)|^{p_{1}}|v(\tau)|^{q_{1}}\right\|_{\eta_{1}} d \tau d s .
\end{aligned}
$$


From (3.13), exist $w^{\prime}, \eta^{\prime}$ satisfying

$$
\max \left\{\frac{p_{1}}{r_{1}}+\frac{q_{1}}{r_{2}}-\frac{2}{N}, \frac{p_{1}}{\eta_{1}}+\frac{q_{1}}{w_{1}}\right\}<\frac{p_{1}}{\eta^{\prime}}+\frac{q_{1}}{w^{\prime}}<\min \left\{1, \frac{1}{\eta_{1}}+\frac{2}{N}, \frac{p_{1}}{r_{1}}+\frac{q_{1}}{r_{2}}\right\}
$$

Note that $0<\frac{N}{2}\left(\frac{p_{1}}{\eta^{\prime}}+\frac{q_{1}}{w^{\prime}}-\frac{1}{\eta_{1}}\right)<1,0<\frac{N}{2}\left(\left(\frac{p_{1}}{r_{1}}+\frac{q_{1}}{r_{2}}\right)-\left(\frac{p_{1}}{\eta^{\prime}}+\frac{q_{1}}{w^{\prime}}\right)\right)<1$ and

$$
2-\gamma_{1}+\frac{N}{2}\left(\frac{1}{r_{1}}-\frac{1}{\eta_{1}}\right)-\frac{N}{2}\left(\frac{p_{1}}{\eta^{\prime}}+\frac{q_{1}}{w^{\prime}}-\frac{1}{\eta_{1}}\right)-\frac{N}{2}\left(\left(\frac{p_{1}}{r_{1}}+\frac{q_{1}}{r_{2}}\right)-\left(\frac{p_{1}}{\eta^{\prime}}+\frac{q_{1}}{w^{\prime}}\right)\right)=0 .
$$

From (3.10) (replacing $w$ by $w^{\prime}$ ) and thanks to lemma 2.5, lemma 2.4 and (2.3), we have

$$
\begin{aligned}
&\|u(t, .)\|_{\eta_{1}} \leq C(1+t)^{-\frac{N}{2}\left(\frac{1}{r_{1}}-\frac{1}{\eta_{1}}\right)}\left\|\left(\frac{1}{2} u_{0}+u_{1}\right)\right\|_{r_{1}} \\
&+C(1+t)^{-\frac{N}{2}\left(\frac{1}{r_{1}}-\frac{1}{\eta_{1}}\right)}\left(\left\|u_{1}\right\|_{L^{\eta_{1}}}+\left\|u_{0}\right\|_{L^{\eta_{1}}}\right)+C(1+t)^{-\frac{N}{2}\left(\frac{1}{r_{1}}-\frac{1}{\eta_{1}}\right)}\left\|u_{0}\right\|_{W^{1, \eta_{1}}} \\
&+\int_{0}^{t}(1+t-s)^{-\frac{N}{2}\left(\frac{p_{1}}{\eta^{\prime}}+\frac{q_{1}}{w^{\prime}}-\frac{1}{\eta_{1}}\right)} \int_{0}^{s}(s-\tau)^{-\gamma_{1}}\|u(\tau, .)\|_{\eta^{\prime}}^{p_{1}}\|v(\tau, .)\|_{w^{\prime}}^{q_{1}} d \tau d s \\
&+\int_{0}^{t} e^{-\frac{t-s}{2}}(t-s) \int_{0}^{s}(s-\tau)^{-\gamma_{1}}\|u(\tau, .)\|_{\eta_{1}}^{p_{1}\left(1-\theta^{\prime}\right)}\|v(\tau, .)\|_{w_{1}}^{q_{1}\left(1-\theta^{\prime}\right)}\|u(\tau, .)\|_{\infty}^{p_{1} \theta^{\prime}} \\
&\|v(\tau, .)\|_{\infty}^{q_{1} \theta^{\prime}} d \tau d s .
\end{aligned}
$$

with $1-\theta^{\prime}=\frac{1}{\eta_{1}}\left(\frac{p_{1}}{\eta_{1}}+\frac{q_{1}}{w_{1}}\right)^{-1}$. Combining the last inequality with (4.1), (4.2) and (4.11), we obtain

$$
\begin{aligned}
& \left.\|u(t, .)\|_{\eta_{1}} \leq C(1+t)^{-\frac{N}{2}\left(\frac{1}{r_{1}}-\frac{1}{\eta_{1}}\right)}\left(\left\|u_{0}\right\|_{r_{1}}+\| u_{1}\right) \|_{r_{1}}\right) \\
& +C(1+t)^{-\frac{N}{2}\left(\frac{1}{r_{1}}-\frac{1}{\eta_{1}}\right)}\left(\left\|u_{1}\right\|_{L^{\eta_{1}}}+\left\|u_{0}\right\|_{L^{\eta_{1}}}\right)+C(1+t)^{-\frac{N}{2}\left(\frac{1}{r_{1}}-\frac{1}{\eta_{1}}\right)}\left\|u_{0}\right\|_{W^{1, \eta_{1}}} \\
& +\left[\sup _{s \in(0, t)} \varphi(s)\right]^{p_{1}}\left[\sup _{s \in(0, t)} \psi(s)\right]^{q_{1}} \\
& \times \int_{0}^{t}(1+t-s)^{-\frac{N}{2}\left(\frac{p_{1}}{\eta^{\prime}}+\frac{q_{1}}{w^{\prime}}-\frac{1}{\eta_{1}}\right)} \int_{0}^{s}(s-\tau)^{-\gamma_{1}}(1+\tau)^{-\frac{N}{2}\left(\frac{p_{1}}{r_{1}}+\frac{q_{1}}{r_{2}}-\left(\frac{p_{1}}{\eta^{\prime}}+\frac{q_{1}}{w^{\prime}}\right)\right)} d \tau d s \\
& +\int_{0}^{t} e^{-\frac{t-s}{2}}(t-s) \int_{0}^{s}(s-\tau)^{-\gamma_{1}}(1+\tau)^{-\left(\alpha p_{1}+\beta q_{1}\right) \theta^{\prime}-\frac{N}{2}\left(\frac{p_{1}}{r_{1}}+\frac{q_{1}}{r_{2}}-\left(\frac{p_{1}}{\eta_{1}}+\frac{q_{1}}{w_{1}}\right)\right)\left(1-\theta^{\prime}\right)} d \tau d s \\
& \times\left[\sup _{s \in(0, t)} \varphi(s)\right]^{p_{1}}\left[\sup _{s \in(0, t)} \psi(s)\right]^{q_{1}}
\end{aligned}
$$


Multiplying both sides of $(5.10)$ by $(1+t)^{\frac{N}{2}\left(\frac{1}{r_{1}}-\frac{1}{\eta_{1}}\right)}$, we find

$$
\begin{aligned}
& (1+t)^{\frac{N}{2}\left(\frac{1}{r_{1}}-\frac{1}{\eta_{1}}\right)}\|u(t, .)\|_{\eta_{1}} \\
\leq & C\left(\left\|u_{0}\right\|_{W^{1, \eta_{1}}}+\left\|u_{0}\right\|_{L^{r_{1}}}+\left\|u_{1}\right\|_{\eta_{1}}+\left\|u_{1}\right\|_{r_{1}}\right)+\left[\sup _{s \in(0, t)} \varphi(s)\right]^{p_{1}}\left[\sup _{s \in(0, t)} \psi(s)\right]^{q_{1}} \\
& \times(1+t)^{2-\gamma_{1}+\frac{N}{2}\left(\frac{1}{r_{1}}-\frac{1}{\eta_{1}}\right)-\frac{N}{2}\left(\frac{p_{1}}{\eta^{\prime}}+\frac{q_{1}}{w^{\prime}}-\frac{1}{\eta_{1}}\right)-\frac{N}{2}\left(\frac{p_{1}}{r_{1}}+\frac{q_{1}}{r_{2}}-\frac{p_{1}}{\eta^{\prime}}-\frac{q_{1}}{w^{\prime}}\right)} \\
& +\left[\sup _{s \in(0, t)} \varphi(s)\right]^{p_{1}}\left[\sup _{s \in(0, t)} \psi(s)\right]^{q_{1}}(1+t)^{\frac{N}{2}\left(\frac{1}{r_{1}}-\frac{1}{\eta_{1}}\right)} \\
& \times \int_{0}^{t} e^{-\frac{t-s}{2}}(t-s) \int_{0}^{s}(s-\tau)^{-\gamma_{1}}(1+\tau)^{-\left(\alpha p_{1}+\beta q_{1}\right) \theta^{\prime}-\frac{N}{2}\left(\frac{p_{1}}{r_{1}}+\frac{q_{1}}{r_{2}}-\left(\frac{p_{1}}{\eta_{1}}+\frac{q_{1}}{w_{1}}\right)\right)\left(1-\theta^{\prime}\right)} d \tau d s .
\end{aligned}
$$

We have from the definition of $\eta_{1}, w_{1}$ that

$$
-\left(\alpha p_{1}+\beta q_{1}\right) \theta^{\prime}-\frac{N}{2}\left(\frac{p_{1}}{r_{1}}+\frac{q_{1}}{r_{2}}-\left(\frac{p_{1}}{\eta_{1}}+\frac{q_{1}}{w_{1}}\right)\right)\left(1-\theta^{\prime}\right)=-\alpha-\left(p_{1} \mu+q_{1} \lambda\right)\left(1-\theta^{\prime}\right)+\gamma_{1}-1 .
$$

Using the fact $\mu=\lambda \frac{w_{1}}{\eta_{1}}$ and the condition $\mu<\gamma_{1}-\alpha$, to obtain

$$
-\alpha-\left(p_{1} \mu+q_{1} \lambda\right)\left(1-\theta^{\prime}\right)+\gamma_{1}-1>-1
$$

It yields then by the Lemma 2.2 that

$$
\begin{aligned}
(t+1)^{\frac{N}{2}\left(\frac{1}{r_{1}}-\frac{1}{\eta_{1}}\right)\|u(t, .)\|_{\eta_{1}} \leq} & C\left(\left\|u_{0}\right\|_{W^{1, \eta_{1}}}+\left\|u_{0}\right\|_{L^{r_{1}}}+\left\|u_{1}\right\|_{r_{1}}\right)+C\left[\sup _{s \in(0, t)} \varphi(s)\right]^{p_{1}}\left[\sup _{s \in(0, t)} \psi(s)\right]^{q_{1}} \\
& +C(1+t)^{\frac{N}{2}\left(\frac{1}{r_{1}}-\frac{1}{\eta_{1}}\right)} \int_{0}^{t} e^{-\frac{t-s}{2}}(t-s)(1+s)^{-\alpha-\left(p_{1} \mu+q_{1} \lambda\right)\left(1-\theta^{\prime}\right)} d s \\
& \times\left[\sup _{s \in(0, t)} \varphi(s)\right]^{p_{1}}\left[\sup _{s \in(0, t)} \psi(s)\right]^{q_{1}} .
\end{aligned}
$$

From (5.4), we obtain $\frac{N}{2}\left(\frac{1}{r_{1}}-\frac{1}{\eta_{1}}\right)-\alpha-\left(p_{1} \mu+q_{1} \lambda\right)\left(1-\theta^{\prime}\right)=\mu-\lambda \frac{w_{1}}{\eta_{1}}=0$. Therefore, by virtue of the Lemma 2.2 again, we conclude

$$
(t+1)^{\frac{N}{2}\left(\frac{1}{r_{1}}-\frac{1}{\eta_{1}}\right)}\|u(t, .)\|_{\eta_{1}} \leq C\left(\left\|u_{0}\right\|_{W^{1, \eta_{1}}}+\left\|u_{0}\right\|_{L^{r_{1}}}+\left\|u_{1}\right\|_{r_{1}}\right)+C\left[\sup _{s \in(0, t)} \varphi(s)\right]^{p_{1}}\left[\sup _{s \in(0, t)} \psi(s)\right]^{q_{1}} .
$$

Now, we estimate $\|u(t, .)\|_{\infty}$. We have to distinguish two situations.

Either: $N \leq 2$ or $\left(\frac{p_{1}}{r_{1}}+\frac{q_{1}}{r_{2}}<\frac{4}{N}\right.$ and $\left.\frac{p_{1}}{\eta_{1}}+\frac{q_{1}}{w_{1}}<\frac{2}{N}\right)$. From (4.3),(4.6) and (4.7), there exists $w^{\prime \prime}, \eta^{\prime \prime}$ such that

$$
\max \left\{\frac{p_{1}}{r_{1}}+\frac{q_{1}}{r_{2}}-\frac{2}{N}, \frac{p_{1}}{\eta_{1}}+\frac{q_{1}}{w_{1}}\right\}<\frac{p_{1}}{\eta^{\prime \prime}}+\frac{q_{1}}{w^{\prime \prime}}<\min \left\{1, \frac{2}{N}, \frac{p_{1}}{r_{1}}+\frac{q_{1}}{r_{2}}\right\} .
$$


Note that $0<\frac{N}{2}\left(\frac{p_{1}}{\eta^{\prime \prime}}+\frac{q_{1}}{w^{\prime \prime}}\right)<1,0<\frac{p_{1}}{\eta^{\prime \prime}}+\frac{q_{1}}{w^{\prime \prime}}<1$ and $0<\frac{N}{2}\left(\frac{p_{1}}{r_{1}}+\frac{q_{1}}{r_{2}}-\left(\frac{p_{1}}{\eta^{\prime \prime}}+\frac{q_{1}}{w^{\prime \prime}}\right)\right)<1$. Since $w^{\prime \prime} \in\left(r_{2}, w_{1}\right)$, using interpolation inequality as in (4.11), we obtain

$$
\begin{aligned}
\|u(t, .)\|_{\infty} \leq & (t+1)^{-\frac{N}{2 r_{1}}}\left(\left\|u_{0}\right\|_{r_{1}}+\left\|u_{1}\right\|_{r_{1}}\right)+(t+1)^{-\frac{N}{2 r_{1}}}\left(\left\|u_{0}\right\|_{W^{1, \infty}}+\left\|u_{1}\right\|_{\infty}\right) \\
& +\int_{0}^{t}(t-s+1)^{-\frac{N}{2}\left(\frac{p_{1}}{\eta^{\prime \prime}}+\frac{q_{1}}{w^{\prime \prime}}\right)} \int_{0}^{s}(s-\tau)^{-\gamma_{1}}\|u(\tau, .)\|_{\eta^{\prime \prime}}^{p_{1}}\|v(\tau, .)\|_{w^{\prime \prime}}^{q_{1}} d \tau d s \\
& +\int_{0}^{t} e^{-\frac{t-s}{2}}(t-s) \int_{0}^{s}(s-\tau)^{-\gamma_{1}}\|u(\tau, .)\|_{\infty}^{p_{1}}\|v(\tau, .)\|_{\infty}^{q_{1}} d \tau d s .
\end{aligned}
$$

Making use (4.1), (4.2) and (4.11), we get

$$
\begin{aligned}
\|u(t, .)\|_{\infty} \leq & (t+1)^{-\frac{N}{2 r_{1}}}\left(\left\|u_{0}\right\|_{r_{1}}+\left\|u_{1}\right\|_{r_{1}}+\left\|u_{0}\right\|_{W^{1, \infty}}+\left\|u_{1}\right\|_{\infty}\right) \\
& +\left[\sup _{s \in(0, t)} \varphi(s)\right]^{p_{1}}\left[\sup _{s \in(0, t)} \psi(s)\right]_{0}^{q_{1}} \int_{0}^{t}(1+t-s)^{-\frac{N}{2}\left(\frac{p_{1}}{\eta^{\prime \prime}}+\frac{q_{1}}{w^{\prime \prime}}\right)} \\
& \times \int_{0}^{s}(s-\tau)^{-\gamma_{1}}(1+\tau)^{-\frac{N}{2}\left(\frac{p_{1}}{r_{1}}+\frac{q_{1}}{r_{2}}-\left(\frac{p_{1}}{\eta^{\prime \prime}}+\frac{q_{1}}{w^{\prime \prime}}\right)\right)} d \tau d s \\
& +\left[\sup _{s \in(0, t)} \varphi(s)\right]^{p_{1}}\left[\sup _{s \in(0, t)} \psi(s)\right]_{0}^{q_{1}} \int_{0}^{t} e^{-\frac{t-s}{2}}(t-s) \int_{0}^{s}(s-\tau)^{-\gamma_{1}}(1+\tau)^{-\left(\alpha p_{1}+\beta q_{1}\right)} d \tau d s
\end{aligned}
$$

and as $\left(\alpha p_{1}+\beta q_{1}\right)<1$,

$$
\alpha-\frac{N}{2 r_{1}}=\frac{p_{2}-q_{1}-1}{\left(q_{1} q_{2}-\left(p_{1}-1\right)\left(p_{2}-1\right)\right)}<0, \quad \alpha+1-\gamma_{1}-\alpha p_{1}-\beta q_{1}=0,
$$

it follows from $\frac{N}{2}\left[\frac{\left(p_{1}-1\right)}{r_{1}}+\frac{q_{1}}{r_{2}}\right]=2-\gamma_{1}$ that

$$
\begin{gathered}
\alpha+2-\gamma_{1}-\frac{N}{2}\left(\frac{p_{1}}{\eta^{\prime \prime}}+\frac{q_{1}}{w^{\prime \prime}}\right)-\frac{N}{2}\left(\frac{p_{1}}{r_{1}}+\frac{q_{1}}{r_{2}}\right)+\frac{N}{2}\left(\frac{p_{1}}{\eta^{\prime \prime}}+\frac{q_{1}}{w^{\prime \prime}}\right) \\
=\alpha+2-\gamma_{1}-\frac{N p_{1}}{2 r_{1}}-\frac{N q_{1}}{2 r_{2}}<\frac{N}{2 r_{1}}+2-\gamma_{1}-\frac{N p_{1}}{2 r_{1}}-\frac{N q_{1}}{2 r_{2}}=0 .
\end{gathered}
$$

Therefore, we conclude from Lemma 2.2 that

$$
(t+1)^{\alpha}\|u(t, .)\|_{\infty} \leq C\left(\left\|u_{0}\right\|_{r_{1}}+\left\|u_{1}\right\|_{r_{1}}+\left\|u_{0}\right\|_{W^{1, \infty}}+\left\|u_{1}\right\|_{\infty}\right)+C G^{p_{1}+q_{1}}(t) .
$$

Or else $N=3$ and $\left(\frac{p_{1}}{r_{1}}+\frac{q_{1}}{r_{2}} \geq \frac{4}{N}\right.$ or $\left.\frac{p_{1}}{\eta_{1}}+\frac{q_{1}}{w_{1}} \geq \frac{2}{N}\right)$. Then, from (4.3), (4.7) and (4.8), we can choose $w^{\prime \prime}$ such that

$$
\max \left\{\frac{p_{1}}{r_{1}}+\frac{q_{1}}{r_{2}}-\frac{2}{N}, \frac{p_{1}}{\eta_{1}}+\frac{q_{1}}{w_{1}}, \frac{2}{N}\right\}<\frac{p_{1}}{\eta^{\prime \prime}}+\frac{q_{1}}{w^{\prime \prime}}<\min \left\{1, \frac{p_{1}}{r_{1}}+\frac{q_{1}}{r_{2}}\right\} .
$$


Since $w^{\prime \prime} \in\left(r_{2}, w_{1}\right), \eta^{\prime \prime}\left(r_{1}, \eta_{1}\right)$ by interpolation inequality, we get

$$
\begin{aligned}
\|u(t, .)\|_{\infty} \leq & C\left(\left\|u_{0}\right\|_{r_{1}}+\left\|u_{1}\right\|_{r_{1}}+\left\|u_{0}\right\|_{W^{1, \infty}}+\left\|u_{1}\right\|_{\infty}\right) \\
& +\int_{0}^{t}(1+t-s)^{-\frac{N}{2}\left(\frac{p_{1}}{\eta^{\prime \prime}}+\frac{q_{1}}{w^{\prime \prime}}\right)} \int_{0}^{s}(s-\tau)^{-\gamma_{1}}\|u(\tau, .)\|_{\eta^{\prime \prime}}^{p_{1}}\|v(\tau, .)\|_{w^{\prime \prime}}^{q_{1}} d \tau d s \\
& +\int_{0}^{t} e^{-\frac{t-s}{2}}(t-s) \int_{0}^{s}(s-\tau)^{-\gamma_{1}}\|u(\tau, .)\|_{\infty}^{p_{1}}\|v(\tau, .)\|_{\infty}^{q_{1}} d \tau d s . \\
\|u(t, .)\|_{\infty} \leq & C\left(\left\|u_{0}\right\|_{r_{1}}+\left\|u_{1}\right\|_{r_{1}}+\left\|u_{0}\right\|_{W^{1, \infty}}+\left\|u_{1}\right\|_{\infty}\right) \\
& +\left[\sup _{s \in(0, t)} \varphi(s)\right]^{p_{1}}\left[\sup _{s \in(0, t)} \psi(s)\right]^{q_{1}} \int_{0}^{s}(1+t-s)^{-\frac{N}{2}\left(\frac{p_{1}}{\eta^{\prime \prime}}+\frac{q_{1}}{w^{\prime \prime}}\right)} \times \\
& \int_{0}^{s}(s-\tau)^{-\gamma_{1}}(\tau+1)^{-\frac{N}{2}\left(\frac{p_{1}}{r_{1}}+\frac{q_{1}}{r_{2}}-\left(\frac{p_{1}}{\eta^{\prime \prime}}+\frac{q_{1}}{w^{\prime \prime}}\right)\right)} d \tau d s \\
& +\left[\sup _{s \in(0, t)} \varphi(s)\right]^{p_{1}}\left[\sup _{s \in(0, t)} \psi(s)\right]_{0}^{q_{1}} \int_{0}^{t} e^{-\frac{t-s}{2}}(t-s) \int_{0}^{s}(s-\tau)^{-\gamma_{1}}(1+\tau)^{-\left(\alpha p_{1}+\beta q_{1}\right)} d \tau d s .
\end{aligned}
$$

Since $\alpha p_{1}+\beta q_{1}<1$, we observe that

$$
\frac{p_{1}}{r_{1}}+\frac{q_{1}}{r_{2}}-\frac{2}{N}<\frac{p_{1}}{r_{1}}+\frac{q_{1}}{r_{2}}-\frac{2}{N}\left(\alpha p_{1}+q_{1} \beta\right) .
$$

It easy to see from (4.3), (4.7) and (4.8) that

$$
\max \left\{\frac{p_{1}}{\eta_{1}}+\frac{q_{1}}{w_{1}}, \frac{2}{N}\right\}<\frac{p_{1}}{r_{1}}+\frac{q_{1}}{r_{2}}-\frac{2}{N}\left(\alpha p_{1}+q_{1} \beta\right) .
$$

From these facts, we can assume that $\left(\frac{p_{1}}{\eta^{\prime \prime}}+\frac{q_{1}}{w^{\prime \prime}}\right) \leq \frac{p_{1}}{r_{1}}+\frac{q_{1}}{r_{2}}-\frac{2}{N}\left(\alpha p_{1}+q_{1} \beta\right)$. Thus

$$
\begin{aligned}
& \alpha+1-\gamma_{1}-\frac{N}{2}\left(\frac{p_{1}}{r_{1}}-\frac{p_{1}}{\eta^{\prime \prime}}\right)-\frac{N}{2}\left(\frac{q_{1}}{r_{2}}-\frac{q_{1}}{w^{\prime \prime}}\right)= \\
& \alpha p_{1}+q_{1} \beta-\frac{N}{2}\left(\frac{p_{1}}{r_{1}}+\frac{q_{1}}{r_{2}}-\left(\frac{p_{1}}{\eta^{\prime \prime}}+\frac{q_{1}}{w^{\prime \prime}}\right)\right) \leq 0,
\end{aligned}
$$

and therefore, we conclude as in the previous case

$$
(t+1)^{\alpha}\|u(t)\|_{\infty} \leq C\left(\left\|u_{0}\right\|_{r_{1}}+\left\|u_{0}\right\|_{\infty}\right)+C\left[\sup _{s \in(0, t)} \varphi(s)\right]^{p_{1}}\left[\sup _{s \in(0, t)} \psi(s)\right]^{q_{1}} .
$$

A combination of the above estimates yields the inequality

$$
\varphi(t) \leq C\left(\left\|u_{0}\right\|_{r_{1}}+\left\|u_{0}\right\|_{\infty}\right)+C\left[\sup _{s \in(0, t)} \varphi(s)\right]^{p_{1}}\left[\sup _{s \in(0, t)} \psi(s)\right]^{q_{1}} .
$$

By analogous computations, we get $\psi(t) \leq C\left(\left\|v_{0}\right\|_{r_{1}}+\left\|v_{0}\right\|_{\infty}\right)+C\left[\sup _{s \in(0, t)} \psi(s)\right]^{p_{2}}\left[\sup _{s \in(0, t)} \varphi(s)\right]^{q_{2}}$. 
Denoting $f(t)=\sup _{s \in(0, t)} \varphi(t)$ and $g(t)=\sup _{s \in(0, t)} \psi(t)$, we get

$$
f(t) \leq C\left(\left\|u_{0}\right\|_{W^{1, r_{1} \cap W^{1, \infty}}}+\left\|u_{1}\right\|_{L^{r_{1} \cap L^{\infty}}}\right)+C f(t)^{p_{1}} g(t)^{q_{1}}
$$

$$
g(t) \leq C\left(\left\|v_{0}\right\|_{W^{1, r_{2}} \cap W^{1, \infty}}+\left\|v_{1}\right\|_{L^{r_{2} \cap L^{\infty}}}\right)+C g(t)^{p_{2}} f(t)^{q_{2}} .
$$

We now define $h(t):=f(t)+g(t)$. Taking into account (4.15), (4.16) reads

$$
\begin{aligned}
& h(t) \leq C\left(A+h^{p_{1}+q_{1}}(t)+h^{p_{2}+q_{2}}(t)\right), \\
& \leq C\left(\varepsilon+h^{p_{1}+q_{1}}(t)+h^{p_{2}+q_{2}}(t)\right), \forall t \in\left[0, T_{\max }\right),
\end{aligned}
$$

where $C$ is positive constant independent of $t$ and $A=\left\|u_{0}\right\|_{W^{1, r_{1} \cap W^{1, \infty}}}+\left\|u_{1}\right\|_{L^{r_{1} \cap L^{\infty}}}+\left\|v_{0}\right\|_{W^{1, r_{2} \cap W^{1, \infty}}}+$ $\left\|v_{1}\right\|_{L^{r_{2} \cap L^{\infty}}}$. By using this estimate and standard arguments as in [32], it then follows that $h(t) \leq C \varepsilon, \forall$ $t \in\left[0, T_{\max }\right)$. Hence $f(t) \leq C \varepsilon, g(t) \leq C \varepsilon, \forall t \in\left[0, T_{\max }\right)$.

Now, we show the global existence result in the energy space. We define $H(t)=\|u(t)\|_{L^{1}}+\|v(t)\|_{L^{1}}$ for all $t \in\left[0, T_{\max }\right)$. Making use of Lemma 2.4 and Lemma 2.5, it is deduced from (3.10) that, for all $t \in\left[0, T_{\max }\right)$,

$$
\left\{\begin{array}{l}
\|u(t)\|_{L^{1}} \leq C\left(\left\|u_{0}\right\|_{W^{1,1}}+\left\|u_{1}\right\|_{L^{1}}\right)+\int_{0}^{t} \int_{0}^{s}(s-\tau)^{-\gamma_{1}}\|u(\tau)\|_{\infty}^{p_{1}}\|v(\tau)\|_{q_{1}}^{q_{1}} d \tau d s, \\
\|v(t)\|_{L^{1}} \leq C\left(\left\|v_{0}\right\|_{W^{1,1}}+\left\|v_{1}\right\|_{L^{1}}\right)+\int_{0}^{s} \int_{0}^{s}(s-\tau)^{-\gamma_{2}}\|v(\tau)\|_{\infty}^{p_{2}}\|u(\tau)\|_{q_{2}}^{q_{2}} d \tau d s
\end{array}\right.
$$

Adding the two inequalities of (5.13) and using the $L^{\infty}$-estimates of $u$ and $v$, we have that

$$
\begin{aligned}
H(t) & \leq C\left(\left\|u_{0}\right\|_{W^{1,1}}+\left\|u_{1}\right\|_{L^{1}}+\left\|v_{0}\right\|_{W^{1,1}}+\left\|v_{1}\right\|_{L^{1}}\right) \\
& +C \int_{0}^{t} \int_{0}^{s}(s-\tau)^{-\gamma_{1}}(1+\tau)^{-\alpha p_{1}-\beta\left(q_{1}-1\right)}\|v(\tau)\|_{1} d \tau d s \\
& +C \int_{0}^{t} \int_{0}^{s}(s-\tau)^{-\gamma_{2}}(1+\tau)^{-\beta p_{2}-\alpha\left(q_{2}-1\right)}\|u(\tau)\|_{1} d \tau d d s \\
& \leq C \varepsilon+C \int_{0}^{t} \int_{0}^{s}\left((s-\tau)^{-\gamma_{1}}(1+\tau)^{-\alpha p_{1}-\beta\left(q_{1}-1\right)}+(s-\tau)^{-\gamma_{2}}(1+\tau)^{-\beta p_{2}-\alpha\left(q_{2}-1\right)}\right) H(\tau) d \tau d s \\
& \leq C \varepsilon+C \int_{0}^{t}\left((1+s)^{\beta-\alpha}+(1+s)^{\alpha-\beta}\right) \sup _{0 \leq \tau \leq s} H(\tau) d s
\end{aligned}
$$

From (5.14), and Gronwall's inequality, we get that $H(t) \leq C \varepsilon \exp C\left(t^{1+\beta-\alpha}+t^{1+\alpha-\beta}\right)$ for all $t \in$ $\left[0, T_{\max }\right)$. Therefore, we have that $(u, v) \in\left\{C\left(\left[0, T_{\max }\right) ; L^{1}\left(\mathbb{R}^{N}\right)\right\}^{2}, N=1,2,3\right.$, so for any $r \geq 1$, the solution $(u, v)$ satisfies 


$$
\begin{aligned}
\|u(t)\|_{L^{r}} & \leq C^{\frac{1}{r}} \varepsilon^{\frac{1}{r}} e^{C\left(t^{1+\beta-\alpha}+t^{1+\alpha-\beta}\right) / r}(1+t)^{-(r-1) \alpha / r}, \\
\|v(t)\|_{L^{r}} & \leq C^{\frac{1}{r}} \varepsilon^{\frac{1}{r}} e^{C\left(t^{1+\beta-\alpha}+t^{1+\alpha-\beta}\right) / r}(1+t)^{-(r-1) \beta / r},
\end{aligned}
$$

for all $t \in\left[0, T_{\max }\right)$.

Now, let $D=\left(\partial_{t}, \nabla\right)$,

$$
u_{L}(t)=K_{1}(t)\left(\frac{1}{2} u_{0}+u_{1}\right)+K_{0}(t) u_{0}, v_{L}(t)=K_{1}(t)\left(\frac{1}{2} v_{0}+v_{1}\right)+K_{0}(t) v_{0} .
$$

From Lemma 2.3 with $m=1$, we see that

$$
\left\{\begin{array}{l}
\left\|D u_{L}(t)\right\| \leq C(1+t)^{-N / 2-1 / 2} I_{0, u},\left\|D v_{L}(t)\right\| \leq C(1+t)^{-N / 2-1 / 2} I_{0, v} \\
\left\|D K_{1}(t-s) * J_{0 \mid s}\left(|v(s)|^{p}\right)\right\| \leq C(1+t-s)^{-N / 2-1 / 2}\left[J_{0 \mid s}^{1-\gamma_{1}}\left(\left\||u(s)|^{p_{1}}|v(s)|^{q_{1}}\right\|_{2}\right)\right. \\
\left.+J_{0 \mid s}^{1-\gamma_{1}}\left(\left\||u(s)|^{p_{1}}|v(s)|^{q_{1}}\right\|_{1}\right)\right] \\
\left\|D K_{1}(t-s) * J_{0 \mid s}\left(|u(s)|^{q}\right)\right\| \leq C(1+t-s)^{-N / 2-1 / 2}\left[J_{0 \mid s}^{1-\gamma_{2}}\left(\left\||v(s)|^{p_{2}}|u(s)|^{q_{2}}\right\|_{2}\right)\right. \\
\left.+J_{0 \mid s}^{1-\gamma_{2}}\left(\left\||v(s)|^{p_{2}}|u(s)|^{q_{2}}\right\|_{1}\right)\right]
\end{array}\right.
$$

where

$$
\left\{\begin{array}{c}
I_{0, u}:=\left\|u_{0}\right\|_{1}+\left\|u_{0}\right\|_{H^{1}}+\left\|u_{1}\right\|_{1}+\left\|u_{1}\right\|_{H^{1}}, \\
I_{0, v}:=\left\|v_{0}\right\|_{1}+\left\|v_{0}\right\|_{H^{1}}+\left\|v_{1}\right\|_{1}+\left\|v_{1}\right\|_{H^{1}} .
\end{array}\right.
$$

Then by use of (5.15) and (5.16) and choosing $\varepsilon \ll 1$, we deduce from (3.10) that

$$
\begin{aligned}
& \|D u(t)\| \leq\left\|D u_{L}(t)\right\|+\int_{0}^{t}\left\|D K_{1}(t-s) * J_{0 \mid s}^{1-\gamma_{1}}\left(|v(s)|^{p}\right)\right\| d s \\
& \leq C(1+t)^{-N / 2-1 / 2} I_{0, u}+C \int_{0}^{t}(1+t-s)^{-N / 2-1 / 2}\left[J_{0 \mid s}^{1-\gamma_{1}}\left(\left\||u(s)|^{p_{1}}|v(s)|^{q_{1}}\right\|_{2}\right)\right. \\
& \left.+J_{0 \mid s}^{1-\gamma_{1}}\left(\left\||u(s)|^{p_{1}}|v(s)|^{q_{1}}\right\|_{1}\right)\right]_{d s}^{t} \\
& \leq C \varepsilon+C \int_{0}^{t}(1+t-s)^{-N / 2-1 / 2} \int_{0}^{s}(s-\tau)^{-\gamma_{1}}\|u\|_{\infty}^{p_{1}}\left(\|v(\tau)\|_{2 q_{1}}^{q_{1}}+\|v(\tau)\|_{q_{1}}^{q_{1}}\right) d \tau d s \\
& \leq C \varepsilon+C \int_{0}^{s}(1+t-s)^{-N / 2-1 / 2} \\
& \times \int_{0}^{s}(s-\tau)^{-\gamma_{1}}\left(\varepsilon^{\frac{1}{2}} e^{C\left(\tau^{1+\beta-\alpha}+\tau^{1+\alpha-\beta}\right) / 2}(1+\tau)^{-\left(2 p_{1}-1\right) \beta / 2}+\varepsilon e^{C\left(\tau^{1+\beta-\alpha}+\tau^{1+\alpha-\beta}\right)}(1+\tau)^{-\left(p_{1}-1\right) \beta}\right) d \tau d s \\
& \leq C\left(1+e^{C\left(t^{1+\beta-\alpha}+t^{1+\alpha-\beta}\right)}(1+t)^{\beta}\right) \sqrt{\varepsilon}, \forall t \in\left[0, T_{\max }\right),
\end{aligned}
$$

where $C$ is positive constant independent of $t$. Similarly

$$
\|D v(t)\| \leq C\left(1+e^{C\left(t^{1+\beta-\alpha}+t^{1+\alpha-\beta}\right)}(1+t)^{\alpha}\right) \sqrt{\varepsilon}, \forall t \in\left[0, T_{\max }\right),
$$

which completes the proof of the Theorem. 


\section{References}

[1] B. de Andrade and A. Viana, On a fractional reaction-diffusion equation, Z. Angew. Math. Phys. 68 (2017), no. 3, Art. 59, $11 \mathrm{pp}$.

[2] D. Andreucci, New results on the Cauchy problem for parabolic systems and equations with strongly nonlinear sources, Manuscripta Math., 77 (1992), 127-159.

[3] T. Cazenave, F. Dickstein, F.B. Weissler, An equation whose Fujita critical exponent is not given by scaling, Nonlinear Anal. 68 (2008) 862-874.

[4] M. Berbiche, Existence and blow-up of solutions for damped ave system with nonlinear memory, Applicable Analysis V. 94, (2015), 2535-2564.

[5] M. Berbiche, Asymptotically self-similar global solutions of a damped wave equation with nonlinear memory, Asymptotic Analysis, vol. 82, no. 3-4, 2013, pp. 315-330

[6] M. Berbiche and A. Hakem, Finite Time Blow-Up of Solutions for Damped Wave Equation with Nonlinear Memory, Commun. Math. Anal. vol 14, Number 1 (2013), 72-84.

[7] D. BLANCHARD AND H. GHIDOUCHE, A nonlinear system for irreversible phase changes, European J. Appl. Math., 1 (1990), pp. 91-100.

[8] S. Cui, Local and global existence of solutions to semilinear parabolic initial value problems, Nonlinear Anal. 43 (2001). 293-323.

[9] L. Debnath, D. Bhatta, Integral Transforms and Their Applications, Taylor and Francis group, 2007.

[10] M. Escobedo, M.A. Herrero, Boundedness and blow up for a semilinear reaction-diffusion system, J. Differential Equations 89 (1991) 176-202.

[11] H. ENGLER, On some parabolic integro-differential equations: existence and asymptotics of solutions, In: Proceedings of the international conference on Equadiff 1982, Würzburg, Lecture notes in mathematics, 1017 (1983), pp. $161-167$.

[12] M. Escobedo,H.A. Levine, Critical blow up and global existence numbers for a weakly coupled system of reactiondiffusion equations, Arch. Rational Mech. Anal. 129 (1995) 47-100.

[13] G. T. HABETLER AND R. L. SCHIFFMAN, A finite difference method for analyzing the compression of poroviscoelastic media, Computing, 6 (1970), pp. 342-348.

[14] H. Fujita, On the blowing up of solutions of the problem for $u_{t}=\Delta u+u^{1+\alpha}$, J. Fac. Sci. Univ. Tokyo 13 (1966) $109-124$.

[15] T. Hosono, T. Ogawa, Large time behavior and $L^{p}-L^{q}$ estimate of solutions of 2-dimensional nonlinear damped wave equations, J. Differential Equations 203 (2004) 82-118.

[16] R. Ikehata, Y. Miyaoka, T. Nakatake, Decays estimates of solutions for dissipative wave equation in $\mathbb{R}^{n}$ with lower power nonlinearities, J. Math. Soc. Japan 56 (2004) 365-373.

[17] R. Ikehata, K. Tanizawa, Global existence for solutions for semilinear damped wave equation in $R^{N}$ with noncompactly supported initial data, Nonlinear. Anal. 61 (2005) 1189-1208.

[18] G. Karch, Self similar profiles in large time asymptotics of solutions to damped wave equations, Studia Math. 143 (2000) 175-197.

[19] W. E. KASTENBERG, Space dependent reactor kinetics with positive feed-back, Nukleonik, 11 (1968), pp. 126-130.

[20] S. Kawashima, M. Nakao, K. Ono, On the decay property of solutions to the Cauchy problem of the semilinear wave equation with a dissipative term, J. Math. Soc. Japan 47 (1995) 617-653.

[21] A.A. Kilbas, H.M. Srivastava, J.J. Trujillo, Theory and Applications of Fractional Differential Equations, 2006. 
[22] M. Loayza a, and I. Quinteiro, A nonlocal in time parabolic system whose Fujita critical exponent is not given by scaling, J. Math. Anal. Appl. 374 (2011) 615-632.

[23] A. Matsumura, On the asymptotic behavior of solutions of semi-linear wave equations, Publ. RIMS Kyoto Univ. 121 (1976) 169-189.

[24] Li T. T., Zhou Y., Breakdown of solutions to $\square u+u_{t}=|u|^{1+\alpha}$, Discrete Contin Dyn Syst, 1, pp.503-520, 1995.

[25] T. Narazaki, $L^{p}-L^{q}$ estimates for damped wave equations and their applications to semi-linear problem, J. Math. Soc. Japan 56 (2004) 585-626.

[26] K. Marcati, P. Nishihara, The $L^{p}-L^{q}$ estimates of solutions to one-dimensional damped wave equations and their application to compressible flow through porous media, J. Differential Equations 191 (2003) 445-469.

[27] K. Nishihara, $L^{p}-L^{q}$ estimates of solutions to the damped wave equation in 3-dimensional space and their application, Math. Z. 244 (2003) 631-649.

[28] T. Ogawa, H. Takeda, Global Existence of solutions for a system of nonlinear damped wave equations, Differential Integral Equations 23 (2010) 635-657.

[29] B. G. PACHPATTE, On a nonlinear diffusion system arising in reactor dynamics, J. Math. Anal. Appl., 94 (1983), pp. 501-508.

[30] C. V. PAO, Bifurcation analysis of a nonlinear diffusion system in reactor dynamics, Appl. Anal., 9 (1979), pp. 107125.

[31] G. Ponce, Global existence of small solutions to a class of nonlinear evolution equations, Nonlinear Anal., (9) (1985), 399-418.

[32] R. Racke, Lectures on nonlinear evolution equations. Initial value problems. Aspects of mathematics, E19. Friedrich Vieweg and Sohn: Braunschweig, Wiesbaden, 1992.

[33] F. Sun, M. Wang, Existence and nonexistence of global solutions for a nonlinear hyperbolic system with damping, Nonlinear Anal. 66 (12) (2007) 2889-2910.

[34] S. Snoussi, S. Tayachi, Global existence, asymptotic behavior and self-similar solutions for a class of semilinear parabolic systems, Nonlinear Analysis, 48 (2002), 13-35.

[35] H. Takeda, obal existence and Nonexistence of solutions for a system of nonlinear damped wave equations, J. Math. Anal. Appl. 360 (2009) 631-650.

[36] G. Todorova, B. Yordanov, Critical exponent for a nonlinear wave equation with damping, J. Differential Equations (174) (2001) 464-489.

[37] Y. YAMADA, On a certain class of semilinear Volterra diffusion equations, J. Math. Anal. Appl., 88 (1982), pp. 433-457.

[38] Y. YAMADA, Asymptotic stability for some systems of semilinear Volterra diffusion equations, J. Differ. Equations, 52 (1984), pp. 295-326.

[39] Han Yang, Jianli Shi and Shihui Zhu, Global existence of solutions for damped wave equations with nonlinear memory, Applicable Analysis to appear (2012).

[40] Yong qiang $\mathrm{Xu}$, Existence and nonexistence of global solutions for a damped hyperbolic system with nonlinear memory, Chinese Journal of Contemporary Mathematics (29), Number 6, (2012) 1-24.

[41] M W, SHENGJIA Li, L. Lu, Critical exponent for a damped wave system with fractional integral, Electronic Journal of Differential Equations, Vol. 2015 (2015), No. 211, pp. 1-10.

[42] Y Li, C Mu, L Wang, Lifespan and a new critical exponent for a nonlocal parabolic equation with slowly decay initial values, Applicable Analysis Vol. 92, (12) 2013, 2618-2629. 
[43] Qi S. Zhang, A blow up result for a nonlinear wave equation with damping: the critical case, C. R. Acad. Sci. Paris 333 (2) (2001) 109-114.

[44] Q.-G. Zhang and H.-R. Sun, The blow-up and global existence of solutions of Cauchy problems for a time fractional diffusion equation, Topol. Methods Nonlinear Anal. 46 (2015), no. 1, 69-92. 